\title{
Anfíbios do Estado de São Paulo, Brasil: conhecimento atual e perspectivas
}

\author{
Denise de Cerqueira Rossa-Feres ${ }^{1,6}$, Ricardo Jannini Sawaya ${ }^{2}$, Julián Faivovich ${ }^{3}$, \\ João Gabriel Ribeiro Giovanelli, Cinthia Aguirre Brasileiro ${ }^{2}$, Luis Schiesari ${ }^{5}$, \\ João Alexandrino ${ }^{2}$ \& Célio Fernando Baptista Haddad ${ }^{4}$
}

${ }^{1}$ Departamento de Zoologia e Botânica, Universidade Estadual Paulista - UNESP, R. Cristovão Colombo, n. 2265, Jardim Nazareth, CEP 15054-000, São José do Rio Preto, SP, Brasil ${ }^{2}$ Departamento de Ciências Biológicas, Universidade Federal de São Paulo - UNIFESP, R. Prof. Artur Riedel, n. 275, Eldorado, CEP 09972-270, Diadema, SP, Brasil ${ }^{3}$ División Herpetología, Museo Argentino de Ciencias Naturales - CONICET, R. Angel Gallardo, n. 470, C1405DJR, Buenos Aires, Argentina ${ }^{4}$ Departamento de Zoologia, Instituto de Biociências, Universidade Estadual Paulista - UNESP, Av. 24-A, n. 1515, Bela Vista, CEP 13506-970, Rio Claro, SP, Brasil

${ }^{5}$ Escola de Artes, Ciências e Humanidades, Gestão Ambiental, Universidade de São Paulo - USP, Av. Arlindo Bétio, n. 1000, Parque Ecológico do Tietê, CEP 03828-080, São Paulo, SP, Brasil

${ }^{6}$ Autor para correspondência: Denise de Cerqueira Rossa-Feres, e-mail: denise@ibilce.unesp.br

ROSSA-FERES, D.C., SAWAYA, R.J., FAIVOVICH, J., GIOVANELLI, J.G.R., BRASILEIRO, C.A., SCHIESARI, L., ALEXANDRINO, J. \& HADDAD, C.F.B. Amphibians of São Paulo State, Brazil: state-ofart and perspectives. Biota Neotrop. 11(1a): http://www.biotaneotropica.org.br/v11n1a/en/abstract?inventory + bn0041101a2011

Abstract: The last list of species of the state of São Paulo State was updated and totaled 236 species of amphibians, 230 of which are anurans and six are caecilians. Bokermannohyla gouveai and Sphaenorhynchus surdus were removed from this list, because they did not occur in the State of São Paulo. The number of anuran species recorded comprise $27 \%$ of the species richness of the country and an increase by $31 \%$ in the number of species recorded for the state since 1998. Thus, despite the State of São Paulo be the Brazilian region where the anurans have been most studied, these data show that the number of known species tends to increase in the next years. We have identified two major geographical gaps of inventory: the southwest of the state, especially in the Paranapanema river basin and the northeast region, mainly at the border between the States of Minas Gerais and São Paulo. Although both have been sampled recently, information is still lacking. The current state of knowledge and perspectives in the areas such as taxonomy, systematics, ecology and conservation are evaluated.

Keywords: Amphibia, Anura, Gymnophiona, biodiversity, State of São Paulo, conservation, inventory, BIOTA/ FAPESP program.

Number of species: In the world: 6,638 (Frost 2010), in Brazil: 875 (Sociedade Brasileira de Herpetologia 2010), estimated in São Paulo State: 250 (Araújo et al. 2009).

ROSSA-FERES, D.C., SAWAYA, R.J., FAIVOVICH, J., GIOVANELLI, J.G.R., BRASILEIRO, C.A., SCHIESARI, L., ALEXANDRINO, J. \& HADDAD, C.F.B. Anfíbios do Estado de São Paulo, Brasil: conhecimento atual e perspectivas. Biota Neotrop. 11(1a): http://www.biotaneotropica.org.br/v11n1a/pt/abstract?inventory+bn004 $1101 \mathrm{a} 2011$

Resumo: A última lista de espécies do Estado de São Paulo foi atualizada, totalizando 236 espécies de anfíbios, das quais 230 são anuros e seis são Gymnophiona. Foram removidos da lista Bokermannohyla gouveai e Sphaenorhynchus surdus por não ocorrerem no Estado de São Paulo. O número de espécies de anuros registrado representa $27 \%$ da riqueza de espécies do país e um aumento de $31 \%$ em relação ao número de espécies registradas para o Estado em 1998, demonstrando que, a despeito do Estado de São Paulo ser a região brasileira onde os anuros foram mais estudados, o número de espécies conhecidas deve aumentar nos próximos anos. Foram evidenciadas duas lacunas geográficas importantes: a região sudoeste do Estado, principalmente na bacia hidrográfica do rio Paranapanema e a região nordeste, principalmente na divisa entre os Estados de Minas Gerais e São Paulo. Apesar de ambas terem sido amostradas recentemente, ainda há carência de informações. O estado atual do conhecimento e perspectivas nas áreas de taxonomia, sistemática, ecologia e conservação são avaliados.

Palavras-chave: Amphibia, Anura, Gymnophiona; biodiversidade, Estado de São Paulo, conservação, inventário, programa BIOTA/FAPESP.

Número de espécies: No mundo: 6.638 (Frost 2010), no Brasil: 875 (Sociedade Brasileira de Herpetologia 2010), estimadas no Estado de São Paulo: 250 (Araújo et al. 2009). 


\section{Introdução}

\section{Classificação geral dos anfíbios}

Três grandes ordens abrigam os anfíbios atuais: Anura, Urodela e Gymnophiona. Na ordem Anura estão os sapos, rãs e pererecas, anfíbios com corpo curto e sem cauda, a maioria com adaptações para o salto; os urodelos incluem as salamandras, anfíbios com cauda e aspecto de lagartos, enquanto na ordem Gymnophiona estão alocados os anfíbios sem patas, com aspecto de pequenas serpentes e hábito geralmente fossorial (Duellman \& Trueb 1994).

Recentemente, a classificação dos anfíbios vem sofrendo alterações significativas, procurando refletir o conhecimento atual sobre suas relações filogenéticas, a partir da intensificação de estudos sobre a sistemática do grupo (e.g., Faivovich et al. 2005, Frost et al. 2006, Hedges et al. 2008, Guayasamin et al. 2008). Essas mudanças envolvem a proposição de novos grupos de espécies, gêneros e famílias, e re-alocações de espécies, gêneros e famílias. Novas mudanças deverão ocorrer em breve, pois esses estudos são recentes e algumas hipóteses filogenéticas atuais estão ainda pouco suportadas.

\section{Ecologia, biologia reprodutiva e distribuição espacial}

A despeito da grande diversidade de modos reprodutivos, envolvendo desde ovos e girinos aquáticos até ovos terrestres com desenvolvimento direto (Duellman \& Trueb 1994, Haddad \& Prado 2005), o ciclo de vida dos anfíbios é fortemente influenciado pela distribuição e abundância de água, normalmente na forma de chuva (McDiarmid 1994). Esses dois aspectos da história natural dos anfíbios promovem processos opostos: por um lado a grande diversidade de modos reprodutivos possibilita que ocupem os mais diversos tipos de hábitat, favorecendo sua distribuição espacial e, por outro lado, a grande dependência de umidade os torna altamente vulneráveis a variações ambientais (e.g., Werner et al. 2007) e, portanto, à extinção local (Lips 1999, Bosch 2003), pois poucos tetrápodes são tão dependentes da umidade como os anfíbios (Duellman \& Trueb 1994). Estudos de longo prazo tem detectado flutuações populacionais naturais de grande amplitude (Blaustein \& Wake 1990, Pechman et al. 1991, Rossa-Feres 1997) mas, atualmente, não há dúvidas sobre a ocorrência de declínio global dos anfíbios (Wake 1991, Bosch 2003, Pounds et al. 2006, 2007). As possíveis causas desse declínio variam desde mudanças climáticas, poluição industrial e por agrotóxicos, introdução de espécies exóticas, até doenças emergentes (Kiesecker et al. 2001, Bosch 2003, Pounds et al. 2006, 2007). Mas a principal ameaça aos anfíbios no Brasil e no mundo é a destruição, degradação e fragmentação de hábitats (Haddad 1998, Silvano \& Segalla 2005, Becker et al. 2007).

Os diferentes modos reprodutivos dos anfíbios são associados a diferentes graus de umidade e disponibilidade de água, e essa relação parece regular sua distribuição espacial e, possivelmente, representa um filtro evolutivo para a distribuição geográfica nos diferentes biomas. Vasconcelos et al. (2010) concluíram que o volume de chuva anual foi a principal variável climática predizendo a riqueza de espécies e o número de modos reprodutivos em 36 localidades brasileiras. A maior riqueza de espécies foi encontrada nas localidades com maior volume anual de chuva e o grau de concentração de chuva foi negativamente correlacionado tanto com a riqueza de espécies quanto com o número de modos reprodutivos. Esse padrão de distribuição da riqueza de espécies é evidente no Estado de São Paulo, onde se pode perceber um gradiente decrescente no número de espécies desde as áreas próximas ao litoral, no domínio da Mata Atlântica (211 espécies; Araújo et al. 2009) onde o clima é mais úmido, para áreas mais ao norte e oeste do estado, no domínio do
Cerrado e da Floresta Estacional (58 espécies; Araújo et al. 2009) onde a estação seca é pronunciada (Santos et al. 2007, Rossa-Feres et al. 2008, Araujo et al. 2009). O número de modos reprodutivos acompanha essa tendência, pois espécies com modos reprodutivos semiterrestres ou terrestres são comuns em áreas com pouca variação sazonal (Haddad \& Prado 2005) e são raras ou ausentes em regiões sazonalmente secas (Vasconcelos \& Rossa-Feres 2005, Zina et al. 2007, Santos et al. 2007, Vasconcelos et al. 2010).

Mesmo que a riqueza de espécies seja moderada em uma localidade com grande umidade, o número de modos reprodutivos tende a ser alto, porque a umidade pode fornecer microhábitats adequados para modos reprodutivos terrestres (Duellman 1988, Hödl 1990). Espécies com modos generalizados ocorrem em todos os biomas, mas são mais numerosas em regiões com clima sazonal (estação seca bem definida), como o Cerrado e a Floresta Estacional (Vasconcelos \& Rossa-Feres 2005, Zina et al. 2007, Brassaloti et al. 2010). Já espécies com modos reprodutivos especializados dependem tanto da disponibilidade de microhábitats úmidos quanto da diversidade de tipos de microhábitats, como encontrado na Mata Atlântica (Haddad \& Prado 2005).

\section{Metodologia}

A primeira compilação das espécies de anfíbios conhecidas para o Estado de São Paulo foi publicada recentemente (Araújo et al. 2009). A amostragem foi realizada nas principais coleções científicas do Estado e a atualização da lista foi efetuada com base na experiência profissional dos autores, em entrevistas com outros herpetológos e na literatura especializada. Além disso, foram utilizadas as informações compiladas durante a atualização da lista de anfíbios ameaçados de extinção no Estado de São Paulo (Garcia et al. 2009), para a qual foram realizadas consultas por meio de questionários e reuniões temáticas.

Para a identificação das regiões com maior número de amostragens de anfíbios, e consequentemente das lacunas geográficas de conhecimento ainda existentes no Estado de São Paulo, foram efetuadas buscas de registros de coletas nas principais coleções científicas do estado. Para isso foram utilizados os dados disponíveis na página eletrônica do SpeciesLink, uma fonte de informação que integra dados sobre biodiversidade disponível em museus, herbários e coleções científicas (SpeciesLink 2010). A opção por usar somente dados eletrônicos se deu pela disponibilização de coordenadas geográficas padronizadas para todos os registros de espécies. Para o Estado de São Paulo estão disponíveis as Coleções "Célio F.B. Haddad, Instituto de Biociências - Universidade Estadual Paulista - campus de Rio Claro (CFBH)", "Coleção de Anfíbios do Departamento de Zoologia e Botânica da Universidade Estadual Paulista - campus de São José do Rio Preto (DZSJRP-Amphibia adults/tadpoles)" e "Coleção de Anfíbios do Museu de Zoologia da UNICAMP (ZUEC- Amphibia)". Apesar da alta representatividade e maior número de registros, a Coleção de Anfíbios do Museu de Zoologia da Universidade de São Paulo (MZUSP) não está disponível na base de dados do SpeciesLink, pois a maior parte de seu acervo precisa ser conferida e determinada com segurança, além de cerca de um terço dos registros ainda não estar digitalizado.

Após a compilação dos registros de coletas nas Coleções, foi possível utilizar o aplicativo DIVA-GIS (Hijmans et al. 2002), que através da interpolação "circular neighborhood" (Cressie 1991), gerou um mapa em formato rasterizado. Este mapa indica as regiões do Estado com maior número de registros de coleta de anfíbios e as regiões menos amostradas, sendo as últimas consideradas como lacunas de conhecimento. 


\section{Resultados e Discussão}

\section{Lista das espécies de anfíbios do Estado de São Paulo}

A lista aqui apresentada (Tabela 1) é uma atualização da apresentada em Araújo et al. (2009). Espécies recentemente descritas ou que sofreram mudanças nomenclaturais, realocação de gênero ou família desde a publicação de Araújo et al. (2009) são destacadas em negrito na tabela, como Cycloramphus jordanensis Heyer, 1983, que foi alocado no gênero Megaelosia (Verdade \& Rodrigues 2008). Foram removidos da lista Bokermannohyla gouveai (Peixoto and Cruz, 1992) e Sphaenorhynchus surdus (Cochran, 1953), que não ocorrem em São Paulo (C.F.B. Haddad e J. Faivovich, obs. pess., Toledo et al. 2007). A lista atual conta com 236 espécies de anfíbios, das quais 230 são anuros pertencentes a 13 famílias e 45 gêneros, e seis espécies são Gymnophiona, pertencentes a uma única família e três gêneros (Tabela 1). Uma análise mais detalhada da lista e da distribuição taxonômica e geográfica das espécies do Estado de São Paulo pode ser encontrada em Araújo et al. (2009).

\section{Principais avanços relacionados ao Programa BIOTA}

O programa BIOTA (http://www.biota.org.br/), financiado pela Fundação de Amparo à Pesquisa do Estado de São Paulo (FAPESP), é certamente uma das maiores iniciativas empreendidas no Brasil e no mundo para o estudo e conservação da biodiversidade (Metzger \& Casatti 2006, Joly et al. 2010). O programa apoia projetos de levantamento florístico e faunístico no Estado de São Paulo, bem como a sistematização desta informação em um banco de dados de amplo acesso (SinBiota, http://sinbiota.cria.org.br/), e possibilita a informatização e disponibilização em rede de 35 grandes coleções científicas (SpeciesLink, http://splink.cria.org.br/). Desta forma, este programa representa uma das maiores contribuições para a definição dos indicadores ecológicos e para a escolha de áreas prioritárias para conservação (Metzger \& Casatti 2006, Joly et al. 2010). Recentemente, utilizando e complementando essas bases de dados, foi produzido um diagnóstico e definidas áreas prioritárias para inventários biológicos, para incremento da conectividade e para conservação no Estado de São Paulo (Rodrigues et al. 2008). Em 13 de março de 2008, a Secretaria de Estado do Meio Ambiente publicou a RESOLUÇÃO SMA-15, estabelecendo que a análise dos pedidos de supressão de vegetação nativa no Estado de São Paulo nos imóveis rurais deverá considerar as categorias de importância para a manutenção e restauração da conectividade biológica, definidas no mapa denominado "Áreas Prioritárias para Incremento para Conectividade" do Projeto BIOTA/FAPESP (D.O. 14/03/08 Seção I - pág.36). Atualmente há quatro decretos governamentais que seguem as orientações do Programa, incluindo uma resolução conjunta das secretarias estaduais do Meio Ambiente e da Agricultura para estabelecer uma lei de zoneamento agro-ecológico, que proíbe a expansão da cana-de-açúcar em áreas consideradas prioritárias para a conservação da biodiversidade e restauração (Resolução SMA $\mathrm{n}^{\circ} 88$, de 19 de dezembro de 2008, Republicada em 26 de março de 2009 - Seção I - pág. 34-35), e o estabelecimento em 31 de março de 2010 de uma área de conservação na restinga de Bertioga (Guaratuba e Itaguaré) [Decreto n ${ }^{\circ}$ 55.661, Diário Oficial - Poder Executivo Seção I São Paulo, 120 (60)].

Especificamente em relação aos anfíbios, o Programa apoiou três Projetos temáticos (http://www.biota.org.br/projeto/index?searc; Apêndice 1), três projetos Jovem Pesquisador (Apêndice 1), vários auxílios regulares a projetos de pesquisa, e bolsas de Iniciação Científica, Mestrado e Doutorado, vinculados ou não a Projeto Temático ou Jovem Pesquisador. As informações sobre distribuição e uso de hábitat pelas espécies de anuros registradas com o desenvolvimento desses projetos foram consideradas no estabelecimento das diretrizes para a conservação da herpetofauna no Estado de São Paulo (Rossa-Feres et al. 2008). Pela primeira vez, a anurofauna de regiões de Floresta Estacional, da Serra da Mantiqueira e da Serra da Bocaina, esta última de grande importância histórica e taxonômica para o grupo (e.g., Lutz 1925a,b, 1929, Cochran 1938, Bokermann 1966), estão sendo sistematicamente inventariadas no Estado de São Paulo (Projetos 1, 2, 4, 5, 6, 10 e 11 - Apêndice 1), superando uma limitação identificada já no início do programa Biota/FAPESP, e eliminando um viés de amostragem direcionado para áreas de Mata Atlântica que limitava qualquer generalização acerca da riqueza de espécies de anfíbios no estado (Haddad 1998, Rossa-Feres et al. 2008).

Atualmente, 236 espécies de anfíbios são conhecidas para o Estado de São Paulo (Rossa-Feres et al. 2008, Araújo et al. 2009, este estudo), o que representa $27 \%$ da riqueza de espécies no país (875 espécies, Sociedade Brasileira de Herpetologia 2010) e um aumento de $31 \%$ em relação ao número de espécies registradas para o estado em 1998 (180 espécies; Haddad 1998). Esse aumento resulta tanto da realização de estudos em localidades que ainda não haviam sido inventariadas, como a Serra da Mantiqueira, Vale do Paraíba, Serra da Bocaina, o extremo nordeste do estado, áreas de Cerrado e ilhas continentais, quanto do aumento no esforço de amostragem em áreas já estudadas (Rossa-Feres et al. 2008). Como resultado, houve um grande aumento no registro de espécies já conhecidas em outros Estados (e.g., Sawaya \& Haddad 2006, Araujo et al. 2007a, b, 2009, Prado et al. 2008, Toledo et al. 2008, Silva et al. 2009, 2010), bem como nas descobertas de novas espécies (Brasileiro et al. 2007a, b, c, Berneck et al. 2008, Toledo 2010, Toledo et al. 2010). Esses resultados corroboram as previsões de cerca de 250 espécies para o estado (Haddad 1998, Rossa-Feres et al. 2008) e demonstram que, apesar da intensificação do esforço de amostragens e da ampliação das áreas inventariadas, e a despeito do Estado de São Paulo ser a região brasileira onde os anuros foram mais estudados (Araújo et al. 2009), o número de espécies conhecidas em São Paulo deve aumentar nos próximos anos, como evidenciado pela análise da curva de acumulação de espécies baseada nos registros existentes até hoje no estado (veja em Araújo et al. 2009).

\section{Principais acervos e grupos de pesquisa no Estado de São Paulo}

Os principais acervos, com os números de exemplares estão relacionados em Araújo et al. (2009). Os principais grupos de pesquisa no estado são apresentados abaixo, pelas respectivas Instituições de Ensino e Pesquisa. O grande aumento no número de grupos, em comparação com os listados há 12 anos (Haddad 1998), explica o grande avanço no conhecimento sobre os anfíbios no estado:

- Instituto Butantan/São Paulo, Laboratório de Ecologia e Evolução: Maria José J. Silva, Selma M. Almeida-Santos citogenética, sistemática, reprodução e evolução de anfíbios.

- Instituto Butantan/São Paulo, Laboratório de Biologia Celular: Carlos A.G.S. Jared, Marta Maria Antoniazzi - biologia e história natural de anfíbios anuros e gimnofionos com ênfase na defesa química através das glândulas cutâneas.

- Instituto Florestal/São Paulo: Cybele O. Araujo - diversidade, distribuição e conservação de anfíbios e répteis de Cerrado e Floresta Estacional do Estado de São Paulo.

- UFABC/ Campus Santo André, Centro de Ciências Naturais e Humanas: Vanessa Kruth Verdade - sistemática, morfologia comparada e conservação de anuros.

- UNESP/Campus de Botucatu, Departamento de Zoologia: Jorge Jim - taxonomia, biologia e ecologia de anuros. 
Rossa-Feres, D.C. et al.

Tabela 1. Anfíbios do Estado de São Paulo, suas distribuições nos dois principais biomas e endemismos no Estado (atualizado de Araújo et al. 2009). As espécies que sofreram mudanças de nomenclatura, realocação de gênero ou família depois de Araújo et al. (2009), são destacadas em negrito. Espécies consideradas endêmicas são aquelas com pequena área de ocorrência conhecida, mas não necessariamente restritas ao Estado de São Paulo.

Table 1. Amphibians of the State of São Paulo, their distributions in two main biomes and endemisms in the state (after Araújo et al. 2009). The species' names that have changed or species that were relocated in other family or genus after Araújo et al. (2009) are in bold. Endemic species are those with narrow distribution area, but not necessarily restricted to São Paulo.

\begin{tabular}{|c|c|c|c|}
\hline Espécies & Mata Atlântica & Cerrado & Espécies endêmicas \\
\hline \multicolumn{4}{|l|}{ GYMNOPHIONA } \\
\hline \multicolumn{4}{|l|}{ Caeciliidae } \\
\hline Luetkenotyphlus brasiliensis (Lütken, 1851) & $\mathrm{X}$ & - & - \\
\hline Microcaecilia supernumeraria Taylor, 1969 & $\mathrm{X}$ & - & - \\
\hline Siphonops annulatus (Mikan, 1820) & $\mathrm{X}$ & - & - \\
\hline Siphonops hardyi Boulenger, 1888 & $\mathrm{X}$ & - & - \\
\hline Siphonops insulanus Ihering, 1911 & $\mathrm{X}$ & - & - \\
\hline Siphonops paulensis Boettger, 1892 & $\mathrm{X}$ & - & - \\
\hline \multicolumn{4}{|l|}{ ANURA } \\
\hline \multicolumn{4}{|l|}{ Brachycephalidae } \\
\hline Brachycephalus ephippium (Spix, 1824) & $\mathrm{X}$ & - & - \\
\hline Brachycephalus hermogenesi (Giaretta \& Sawaya, 1998) & $\mathrm{X}$ & - & - \\
\hline Brachycephalus nodoterga Miranda-Ribeiro, 1920 & $\mathrm{X}$ & - & $\mathrm{X}$ \\
\hline Brachycephalus pitanga Alves, Sawaya, Reis \& Haddad, 2009 & $\mathrm{X}$ & - & $\mathrm{X}$ \\
\hline Brachycephalus vertebralis Pombal, 2001 & $\mathrm{X}$ & - & $\mathrm{X}$ \\
\hline Ischnocnema bolbodactyla (A. Lutz, 1925) & $\mathrm{X}$ & - & - \\
\hline Ischnocnema gehrti (Miranda-Ribeiro, 1926) & $\mathrm{X}$ & - & - \\
\hline Ischnocnema guentheri (Steindachner, 1864) & $\mathrm{X}$ & - & - \\
\hline Ischnocnema hoehnei (B. Lutz, 1959 “1958”) & $\mathrm{X}$ & - & - \\
\hline Ischnocnema holti (Cochran, 1948) & $\mathrm{X}$ & - & $\mathrm{X}$ \\
\hline Ischnocnema juipoca (Sazima \& Cardoso, 1978) & $\mathrm{X}$ & $\mathrm{X}$ & - \\
\hline Ischnocnema lactea (Miranda-Ribeiro, 1923) & $\mathrm{X}$ & - & $\mathrm{X}$ \\
\hline Ischnocnema nigriventris (A. Lutz, 1925) & $\mathrm{X}$ & - & - \\
\hline Ischnocnema parva (Girard, 1853) & $\mathrm{X}$ & - & - \\
\hline Ischnocnema pusilla (Bokermann, 1967) & $\mathrm{X}$ & - & $\mathrm{X}$ \\
\hline Ischnocnema randorum (Heyer, 1985) & $\mathrm{X}$ & - & - \\
\hline Ischnocnema spanios (Heyer, 1985) & $\mathrm{X}$ & - & $\mathrm{X}$ \\
\hline \multicolumn{4}{|l|}{ Bufonidae } \\
\hline Dendrophryniscus brevipollicatus Jiménez de la Espada, 1871 “1870” & $\mathrm{X}$ & - & - \\
\hline Dendrophryniscus leucomystax Izecksohn, 1968 & $\mathrm{X}$ & - & - \\
\hline Melanophryniscus moreirae (Miranda-Ribeiro, 1920) & $\mathrm{X}$ & - & $\mathrm{X}$ \\
\hline Rhinella icterica (Spix, 1824) & $\mathrm{X}$ & - & - \\
\hline Rhinella hoogmoedi Caramaschi \& Pombal, 2006 & $\mathrm{X}$ & - & - \\
\hline Rhinella ornata (Spix, 1824) & $\mathrm{X}$ & - & - \\
\hline Rhinella rubescens (A. Lutz, 1925) & - & $\mathrm{X}$ & - \\
\hline Rhinella schneideri (Werner, 1894) & $\mathrm{X}$ & $\mathrm{X}$ & - \\
\hline Centrolenidae & & - & - \\
\hline Vitreorana eurygnatha (A. Lutz, 1925) & $\mathrm{X}$ & - & - \\
\hline Vitreorana uranoscopa (Müller, 1924) & $\mathrm{X}$ & - & - \\
\hline \multicolumn{4}{|l|}{ Ceratophryidae } \\
\hline Ceratophrys aurita (Raddi, 1823) & $\mathrm{X}$ & - & - \\
\hline \multicolumn{4}{|l|}{ Craugastoridae } \\
\hline Haddadus binotatus (Spix, 1824) & $\mathrm{X}$ & - & - \\
\hline \multicolumn{4}{|l|}{ Cycloramphidae } \\
\hline Cycloramphus acangatan Verdade \& Rodrigues, 2003 & $\mathrm{X}$ & - & - \\
\hline Cycloramphus boraceiensis Heyer, 1983 & $\mathrm{X}$ & - & - \\
\hline Cycloramphus carvalhoi Heyer, 1983 & $\mathrm{X}$ & - & $\mathrm{X}$ \\
\hline Cycloramphus dubius (Miranda-Ribeiro, 1920) & $\mathrm{X}$ & - & - \\
\hline
\end{tabular}


Tabela 1. Continuação...

\begin{tabular}{|c|c|c|c|}
\hline Espécies & Mata Atlântica & Cerrado & Espécies endêmicas \\
\hline Cycloramphus eleutherodactylus (Miranda-Ribeiro, 1920) & $\mathrm{X}$ & - & - \\
\hline Cycloramphus faustoi Brasileiro, Haddad, Sawaya, Sazima 2007 & $\mathrm{X}$ & - & $\mathrm{X}$ \\
\hline Cycloramphus granulosus A. Lutz, 1929 & $\mathrm{X}$ & - & - \\
\hline Cycloramphus izecksohni Heyer, 1983 & $\mathrm{X}$ & - & - \\
\hline Cycloramphus juimirim Haddad \& Sazima, 1989 & $\mathrm{X}$ & - & $\mathrm{X}$ \\
\hline Cycloramphus lutzorum Heyer, 1983 & $\mathrm{X}$ & - & - \\
\hline Cycloramphus semipalmatus (Miranda-Ribeiro, 1920) & $\mathrm{X}$ & - & - \\
\hline Cycloramphus stejnegeri (Noble, 1924) & $\mathrm{X}$ & - & $\mathrm{X}$ \\
\hline Macrogenioglottus alipioi Carvalho, 1946 & $\mathrm{X}$ & - & - \\
\hline Odontophrynus americanus (Duméril \& Bibron, 1841) & $\mathrm{X}$ & $\mathrm{X}$ & - \\
\hline Odontophrynus cultripes Reinhardt \& Lütken, 1861"1862" & - & $\mathrm{X}$ & - \\
\hline Proceratophrys appendiculata (Günther, 1873) & $\mathrm{X}$ & - & - \\
\hline Proceratophrys boiei (Wied-Neuwied, 1824) & $\mathrm{X}$ & - & - \\
\hline Proceratophrys melanopogon (Miranda-Ribeiro, 1926) & $\mathrm{X}$ & - & - \\
\hline Proceratophrys moratoi Jim \& Caramaschi, 1980 & - & $X$ & $\mathrm{X}$ \\
\hline Thoropa miliaris (Spix, 1824) & $X$ & - & - \\
\hline Thoropa petropolitana (Wandolleck, 1907) & $\mathrm{X}$ & - & - \\
\hline Thoropa taophora (Miranda-Ribeiro, 1923) & $\mathrm{X}$ & - & - \\
\hline Zachaenus parvulus (Girard, 1853) & $\mathrm{X}$ & - & - \\
\hline \multicolumn{4}{|l|}{ Hemiphractidae } \\
\hline Flectonotus fissilis (Miranda-Ribeiro, 1920) & $\mathbf{X}$ & - & - \\
\hline Flectonotus goeldii (Boulenger, 1895 “1894”) & $\mathbf{X}$ & - & - \\
\hline Flectonotus ohausi (Wandolleck, 1907) & $\mathbf{X}$ & - & - \\
\hline Gastrotheca albolineata (A. Lutz \& B. Lutz, 1939) & $\mathbf{X}$ & - & - \\
\hline Gastrotheca fissipes (Boulenger, 1888) & $\mathbf{X}$ & - & - \\
\hline Gastrotheca microdiscus (Andersson in Lönnberg \& Andersson, 1910) & $\mathbf{X}$ & - & - \\
\hline \multicolumn{4}{|l|}{ Hylidae } \\
\hline Aparasphenodon bokermanni Pombal, 1993 & $\mathrm{X}$ & - & - \\
\hline Aparasphenodon brunoi Miranda-Ribeiro, 1920 & $\mathrm{X}$ & - & - \\
\hline Aplastodiscus albosignatus (A.Lutz \& B.Lutz, 1938) & $\mathrm{X}$ & - & - \\
\hline Aplastodiscus arildae (Cruz \& Peixoto, 1987 “1985”) & $\mathrm{X}$ & - & - \\
\hline Aplastodiscus callipygius (Cruz \& Peixoto, 1985 “1984”) & $\mathrm{X}$ & - & - \\
\hline Aplastodiscus ehrhardti (Müller, 1924) & $\mathrm{X}$ & - & - \\
\hline Aplastodiscus eugenioi (Carvalho-e-Silva \& Carvalho-e-Silva, 2005) & $\mathrm{X}$ & - & - \\
\hline Aplastodiscus leucopygius (Cruz \& Peixoto, 1985 “1984”) & $\mathrm{X}$ & - & - \\
\hline Aplastodiscus perviridis A. Lutz in B. Lutz, 1950 & $\mathrm{X}$ & $\mathrm{X}$ & - \\
\hline Bokermannohyla ahenea (Napoli \& Caramaschi, 2004) & $X$ & - & - \\
\hline Bokermannohyla astartea (Bokermann, 1967) & $\mathrm{X}$ & - & - \\
\hline Bokermannohyla circumdata (Cope, 1871) & $\mathrm{X}$ & - & - \\
\hline Bokermannohyla claresignata (Lutz \& Lutz, 1939) & $X$ & - & - \\
\hline Bokermannohyla clepsydra (A. Lutz, 1925) & $\mathrm{X}$ & - & - \\
\hline Bokermannohyla hylax (Heyer, 1985) & $\mathrm{X}$ & - & - \\
\hline Bokermannohyla izecksohni (Jim \& Caramaschi, 1979) & $\mathrm{X}$ & - & - \\
\hline Bokermannohyla luctuosa (Pombal \& Haddad, 1993) & $\mathrm{X}$ & - & - \\
\hline Bokermannohyla sazimai (Cardoso \& Andrade, 1983"1982") & - & $\mathrm{X}$ & - \\
\hline Dendropsophus anceps (A. Lutz, 1929) & $\mathrm{X}$ & - & - \\
\hline Dendropsophus berthalutzae (Bokermann, 1962) & $\mathrm{X}$ & - & - \\
\hline Dendropsophus decipiens (A. Lutz, 1925) & $X$ & - & - \\
\hline Dendropsophus elegans (Wied-Neuwied, 1824) & $X$ & - & - \\
\hline Dendropsophus elianeae (Napoli \& Caramaschi, 2000) & $X$ & $X$ & - \\
\hline Dendropsophus giesleri (Mertens, 1950) & $\mathrm{X}$ & - & - \\
\hline Dendropsophus jimi (Napoli \& Caramaschi, 1999) & - & $\mathrm{X}$ & - \\
\hline
\end{tabular}


Tabela 1. Continuação...

\begin{tabular}{|c|c|c|c|}
\hline Espécies & Mata Atlântica & Cerrado & Espécies endêmicas \\
\hline Dendropsophus limai (Bokermann, 1962) & $\mathrm{X}$ & - & - \\
\hline Dendropsophus microps (Peter, 1872) & $\mathrm{X}$ & - & - \\
\hline Dendropsophus minutus (Peters, 1872) & $\mathrm{X}$ & $\mathrm{X}$ & - \\
\hline Dendropsophus nanus (Boulenger, 1889) & $\mathrm{X}$ & $\mathrm{X}$ & - \\
\hline Dendropsophus rhea (Napoli \& Caramaschi, 1999) & - & $\mathrm{X}$ & - \\
\hline Dendropsophus sanborni (Schmidt, 1944) & $\mathrm{X}$ & $\mathrm{X}$ & - \\
\hline Dendropsophus seniculus (Cope, 1868) & $\mathrm{X}$ & - & - \\
\hline Dendropsophus werneri (Cochran, 1952) & $\mathrm{X}$ & - & - \\
\hline Hypsiboas albomarginatus (Spix, 1824) & $\mathrm{X}$ & - & - \\
\hline Hypsiboas albopunctatus (Spix, 1824) & $\mathrm{X}$ & $\mathrm{X}$ & - \\
\hline Hypsiboas bischoffi (Boulenger, 1887) & $\mathrm{X}$ & - & - \\
\hline Hypsiboas caingua (Carrizo, 1991 “1990”) & $\mathrm{X}$ & - & - \\
\hline Hypsiboas caipora Antunes, Faivovich \& Haddad, 2008 & $\mathrm{X}$ & - & - \\
\hline Hypsiboas crepitans (Wied-Neuwied, 1824) & $\mathrm{X}$ & - & - \\
\hline Hypsiboas cymbalum (Bokermann, 1963) & $\mathrm{X}$ & - & $\mathrm{X}$ \\
\hline Hypsiboas faber (Wied-Neuwied, 1821) & $\mathrm{X}$ & $\mathrm{X}$ & - \\
\hline Hypsiboas latistriatus (Caramaschi \& Cruz, 2004) & $\mathrm{X}$ & - & - \\
\hline Hypsiboas lundii (Burmeister, 1856) & $\mathrm{X}$ & $\mathrm{X}$ & - \\
\hline Hypsiboas pardalis (Spix, 1824) & $\mathrm{X}$ & - & - \\
\hline Hypsiboas polytaenius (Cope, 1870) & $\mathrm{X}$ & - & - \\
\hline Hypsiboas prasinus (Burmeister, 1856) & $\mathrm{X}$ & $\mathrm{X}$ & - \\
\hline Hypsiboas punctatus (Schneider, 1799) & - & $\mathrm{X}$ & - \\
\hline Hypsiboas raniceps Cope, 1862 & $\mathrm{X}$ & $\mathrm{X}$ & - \\
\hline Hypsiboas semilineatus (Spix, 1824) & $\mathrm{X}$ & - & - \\
\hline Itapotihyla langsdorffii (Duméril \& Bibron, 1841) & $\mathrm{X}$ & $\mathrm{X}$ & - \\
\hline Phasmahyla cochranae (Bokermann, 1966) & $\mathrm{X}$ & - & - \\
\hline Phasmahyla guttata (A. Lutz, 1924) & $\mathrm{X}$ & - & - \\
\hline Phrynomedusa bokermanni Cruz, 1991 & $\mathrm{X}$ & - & $\mathrm{X}$ \\
\hline Phrynomedusa fimbriata Miranda-Ribeiro, 1923 & $\mathrm{X}$ & - & - \\
\hline Phrynomedusa marginata (Izecksohn \& Cruz, 1976) & $\mathrm{X}$ & - & - \\
\hline Phrynomedusa vanzolinii Cruz, 1991 & $\mathrm{X}$ & - & - \\
\hline Phyllomedusa azurea Cope, 1862 & - & $\mathrm{X}$ & - \\
\hline Phyllomedusa ayeaye (B. Lutz, 1966) & $\mathrm{X}$ & $\mathrm{X}$ & - \\
\hline Phyllomedusa burmeisteri Boulenger, 1882 & $\mathrm{X}$ & - & - \\
\hline Phyllomedusa distincta A. Lutz in B. Lutz, 1950 & $\mathrm{X}$ & - & - \\
\hline Phyllomedusa rohdei Mertens, 1926 & $\mathrm{X}$ & - & - \\
\hline Phyllomedusa tetraploidea Pombal \& Haddad, 1992 & $\mathrm{X}$ & $\mathrm{X}$ & - \\
\hline Pseudis platensis Gallardo, 1961 & - & $\mathrm{X}$ & - \\
\hline Scinax alcatraz (B. Lutz, 1973) & $\mathrm{X}$ & - & $\mathrm{X}$ \\
\hline Scinax alter (B. Lutz, 1973) & $\mathrm{X}$ & - & - \\
\hline Scinax angrensis (B. Lutz, 1973) & $\mathrm{X}$ & - & - \\
\hline Scinax argyreornatus (Miranda-Ribeiro, 1926) & $\mathrm{X}$ & - & - \\
\hline Scinax ariadne (Bokermann, 1967) & $\mathrm{X}$ & - & - \\
\hline Scinax atratus (Peixoto,1989) & $\mathrm{X}$ & - & - \\
\hline Scinax berthae (Barrio, 1962) & $\mathrm{X}$ & $\mathrm{X}$ & - \\
\hline Scinax brieni (De Witte, 1930) & $\mathrm{X}$ & - & - \\
\hline Scinax caldarum (B. Lutz, 1968) & $\mathrm{X}$ & - & - \\
\hline Scinax canastrensis (Cardoso \& Haddad, 1982) & - & $\mathrm{X}$ & - \\
\hline Scinax crospedospilus (A. Lutz, 1925) & $\mathrm{X}$ & - & - \\
\hline Scinax duartei (B. Lutz, 1951) & $\mathrm{X}$ & - & - \\
\hline Scinax eurydice (Bokermann, 1968) & $\mathrm{X}$ & - & - \\
\hline Scinax faivovichi Brasileiro,Oyamaguchi \& Haddad, 2007 & $\mathrm{X}$ & - & - \\
\hline
\end{tabular}


Tabela 1. Continuação...

\begin{tabular}{|c|c|c|c|}
\hline Espécies & Mata Atlântica & Cerrado & Espécies endêmicas \\
\hline Scinax flavoguttatus (A. Lutz \& B. Lutz, 1939) & $\mathrm{X}$ & - & - \\
\hline Scinax fuscomarginatus (A. Lutz, 1925) & $\mathrm{X}$ & $\mathrm{X}$ & - \\
\hline Scinax fuscovarius (A. Lutz, 1925) & $\mathrm{X}$ & $\mathrm{X}$ & - \\
\hline Scinax hayii (Barbour, 1909) & $\mathrm{X}$ & - & - \\
\hline Scinax hiemalis (Haddad \& Pombal, 1987) & $\mathrm{X}$ & - & - \\
\hline Scinax jureia (Pombal \& Gordo, 1991) & $\mathrm{X}$ & - & - \\
\hline Scinax littoralis (Pombal \& Gordo, 1991) & $\mathrm{X}$ & - & - \\
\hline Scinax nasicus (Cope, 1862) & - & $\mathrm{X}$ & - \\
\hline Scinax obtriangulatus (B. Lutz, 1973) & $\mathrm{X}$ & - & - \\
\hline Scinax peixotoi Brasileiro, Haddad, Sawaya \& Martins, 2007 & $\mathrm{X}$ & - & - \\
\hline Scinax perereca Pombal, Haddad \& Kasahara, 1995 & $\mathrm{X}$ & - & - \\
\hline Scinax perpusillus (A. Lutz \& B. Lutz, 1939) & $\mathrm{X}$ & - & - \\
\hline Scinax rizibilis (Bokermann, 1964) & $\mathrm{X}$ & - & - \\
\hline Scinax similis (Cochran, 1952) & $\mathrm{X}$ & $\mathrm{X}$ & - \\
\hline Scinax squalirostris (A. Lutz, 1925) & - & $X$ & - \\
\hline Scinax trapicheiroi (B. Lutz, 1954) & $X$ & - & - \\
\hline Scinax $x$-signatus (Spix, 1824) & $\mathrm{X}$ & $\mathrm{X}$ & - \\
\hline Sphaenorhynchus caramaschii Toledo, Lingnau, Garcia \& Haddad, 2007 & $\mathrm{X}$ & - & - \\
\hline Sphaenorhynchus orophilus (A. Lutz \& B. Lutz, 1938) & $\mathrm{X}$ & - & - \\
\hline Trachycephalus imitatrix (Miranda-Ribeiro, 1926) & $\mathrm{X}$ & - & - \\
\hline Trachycephalus lepidus (Pombal, Haddad \& Cruz, 2003) & $\mathrm{X}$ & - & - \\
\hline Trachycephalus mesophaeus (Hensel, 1867) & $\mathrm{X}$ & - & - \\
\hline Trachycephalus nigromaculatus Tschudi, 1838 & - & $X$ & - \\
\hline Trachycephalus typhonius (Linnaeus 1758) & $\mathbf{X}$ & $\mathbf{X}$ & - \\
\hline \multicolumn{4}{|l|}{ Hylodidae } \\
\hline Crossodactylus caramaschii Bastos \& Pombal, 1995 & $\mathrm{X}$ & - & - \\
\hline Crossodactylus dispar A. Lutz, 1925 & $\mathrm{X}$ & - & - \\
\hline Crossodactylus gaudichaudii Duméril \& Bibron, 1841 & $\mathrm{X}$ & - & - \\
\hline Crossodactylus grandis B. Lutz, 1951 & $\mathrm{X}$ & - & - \\
\hline Hylodes asper (Müller, 1924) & $\mathrm{X}$ & - & - \\
\hline Hylodes cardosoi Lingnau, Canedo \& Pombal Junior, 2008 & $\mathrm{X}$ & - & - \\
\hline Hylodes dactylocinus Pavan, Narvaes \& Rodrigues, 2001 & $X$ & - & $\mathrm{X}$ \\
\hline Hylodes heyeri Haddad, Pombal \& Bastos, 1996 & $\mathrm{X}$ & - & - \\
\hline Hylodes magalhaesi (Bokermann, 1964) & $\mathrm{X}$ & - & $\mathrm{X}$ \\
\hline Hylodes mertensi (Bokermann, 1956) & $\mathrm{X}$ & - & - \\
\hline Hylodes nasus (Lichtenstein, 1823) & $\mathrm{X}$ & - & - \\
\hline Hylodes ornatus (Bokermann, 1967) & $X$ & - & - \\
\hline Hylodes phyllodes Heyer \& Cocroft, 1986 & $\mathrm{X}$ & - & - \\
\hline Hylodes sazimai Haddad \& Pombal, 1995 & $\mathrm{X}$ & - & - \\
\hline Megaelosia bocainensis Giaretta, Bokermann \& Haddad, 1993 & $X$ & - & $X$ \\
\hline Megaelosia boticariana Giaretta \& Aguiar, 1998 & $\mathrm{X}$ & - & $\mathrm{X}$ \\
\hline Megaelosia goeldii (Baumann, 1912) & $X$ & - & - \\
\hline Megaelosia jordanensis (Heyer, 1983) & $\mathbf{X}$ & - & $\mathbf{X}$ \\
\hline Megaelosia massarti (De Witte, 1930) & $\mathrm{X}$ & - & $\mathrm{X}$ \\
\hline \multicolumn{4}{|l|}{ Leiuperidae } \\
\hline Eupemphix nattereri Steindachner, 1863 & - & $\mathrm{X}$ & - \\
\hline Physalaemus atlanticus Haddad \& Sazima, 2004 & $\mathrm{X}$ & - & - \\
\hline Physalaemus barrioi Bokermann, 1967 & $\mathrm{X}$ & - & - \\
\hline Physalaemus bokermanni Cardoso \& Haddad, 1985 & $X$ & - & $\mathrm{X}$ \\
\hline Physalaemus centralis Bokermann, 1962 & $X$ & $X$ & - \\
\hline Physalaemus cuvieri Fitzinger, 1826 & $\mathrm{X}$ & $\mathrm{X}$ & - \\
\hline Physalaemus jordanensis Bokermann, 1967 & $\mathrm{X}$ & - & - \\
\hline
\end{tabular}


Tabela 1. Continuação...

\begin{tabular}{|c|c|c|c|}
\hline Espécies & Mata Atlântica & Cerrado & Espécies endêmicas \\
\hline Physalaemus maculiventris (A. Lutz, 1925) & $\mathrm{X}$ & - & - \\
\hline Physalaemus marmoratus (Reinhardt \& Lütken, 1862 “1861”) & - & $\mathrm{X}$ & - \\
\hline Physalaemus moreirae (Miranda-Ribeiro, 1937) & $\mathrm{X}$ & - & - \\
\hline Physalaemus olfersii (Lichtenstein \& Martens, 1856) & $\mathrm{X}$ & - & - \\
\hline Physalaemus signifer (Girard, 1853) & $\mathrm{X}$ & - & - \\
\hline Physalaemus spiniger (Miranda-Ribeiro, 1926) & $\mathrm{X}$ & - & - \\
\hline Pseudopaludicola falcipes (Hensel, 1867) & - & $\mathrm{X}$ & - \\
\hline $\begin{array}{l}\text { Pseudopaludicola murundu Toledo, Siqueira, Duarte, Veiga-Menoncello, } \\
\text { Recco-Pimentel \& Haddad, } 2010\end{array}$ & - & $\mathbf{X}$ & $\mathbf{X}$ \\
\hline Pseudopaludicola mystacalis (Cope, 1887) & - & $\mathrm{X}$ & - \\
\hline Pseudopaludicola riopiedadensis Mercadal de Barrio \& Barrio, 1994 & - & $\mathrm{X}$ & $\mathrm{X}$ \\
\hline Pseudopaludicola saltica (Cope, 1887$)$ & - & $\mathrm{X}$ & - \\
\hline \multicolumn{4}{|l|}{ Leptodactylidae } \\
\hline Leptodactylus ajurauna Berneck, Costa \& Garcia, 2008 & $\mathrm{X}$ & - & - \\
\hline Leptodactylus bokermanni Heyer, 1973 & $\mathrm{X}$ & - & - \\
\hline Leptodactylus chaquensis Cei, 1950 & $\mathrm{X}$ & $\mathrm{X}$ & - \\
\hline Leptodactylus flavopictus A. Lutz, 1926 & $\mathrm{X}$ & - & - \\
\hline Leptodactylus furnarius Sazima \& Bokermann, 1978 & $\mathrm{X}$ & $\mathrm{X}$ & - \\
\hline Leptodactylus fuscus (Schneider, 1799) & $\mathrm{X}$ & $\mathrm{X}$ & - \\
\hline Leptodactylus jolyi Sazima \& Bokermann, 1978 & $\mathrm{X}$ & $\mathrm{X}$ & - \\
\hline Leptodactylus labyrinthicus (Spix, 1824) & $\mathrm{X}$ & $\mathrm{X}$ & - \\
\hline Leptodactylus latrans (Steffen, 1815) & $\mathbf{X}$ & $\mathbf{X}$ & - \\
\hline Leptodactylus marmoratus (Steindachner, 1867) & $\mathrm{X}$ & - & - \\
\hline Leptodactylus mystaceus (Spix, 1824) & $\mathrm{X}$ & - & - \\
\hline Leptodactylus mystacinus (Burmeister, 1861) & $\mathrm{X}$ & $\mathrm{X}$ & - \\
\hline Leptodactylus notoaktites Heyer, 1978 & $\mathrm{X}$ & - & - \\
\hline Leptodactylus podicipinus (Cope, 1862) & - & $\mathrm{X}$ & - \\
\hline Leptodactylus sertanejo Giaretta \& Costa, 2007 & - & $\mathrm{X}$ & - \\
\hline Leptodactylus syphax Bokermann, 1969 & - & $\mathrm{X}$ & - \\
\hline Paratelmatobius cardosoi Pombal \& Haddad, 1999 & $\mathrm{X}$ & - & - \\
\hline Paratelmatobius gaigeae (Cochran, 1938) & $\mathrm{X}$ & - & $\mathrm{X}$ \\
\hline Paratelmatobius mantiqueira Pombal \& Haddad, 1999 & $\mathrm{X}$ & - & $\mathrm{X}$ \\
\hline Paratelmatobius poecilogaster Giaretta \& Castanho, 1990 & $\mathrm{X}$ & - & - \\
\hline \multicolumn{4}{|l|}{ Microhylidae } \\
\hline Arcovomer passarellii Carvalho, 1954 & $\mathrm{X}$ & - & - \\
\hline Chiasmocleis albopunctata (Boettger, 1885) & - & $\mathrm{X}$ & - \\
\hline Chiasmocleis atlantica Cruz, Caramaschi \& Izecksohn, 1997 & $\mathrm{X}$ & - & - \\
\hline Chiasmocleis carvalhoi Cruz, Caramaschi \& Izecksohn, 1997 & $\mathrm{X}$ & - & - \\
\hline Chiasmocleis leucosticta (Boulenger, 1888) & $X$ & - & - \\
\hline Chiasmocleis mantiqueira Cruz, Feio \& Cassini, 2007 & $\mathrm{X}$ & - & - \\
\hline Dermatonotus muelleri (Boettger, 1885) & - & $X$ & - \\
\hline Elachistocleis bicolor (Guérin-Méneville, 1838) & $\mathrm{X}$ & $X$ & - \\
\hline Elachistocleis cesarii (Miranda-Ribeiro, 1920) & $\mathbf{X}$ & $\mathbf{X}$ & - \\
\hline Elachistocleis ovalis (Schneider, 1799) & $\mathrm{X}$ & $\mathrm{X}$ & - \\
\hline Myersiella microps (Duméril \& Bibron, 1841) & $\mathrm{X}$ & - & - \\
\hline Stereocyclops parkeri (Wettstein, 1934) & $\mathrm{X}$ & - & - \\
\hline \multicolumn{4}{|l|}{ Strabomantidae } \\
\hline Barycholos ternetzi (Miranda-Ribeiro, 1937) & - & $\mathrm{X}$ & - \\
\hline Holoaden luederwaldti Miranda-Ribeiro, 1920 & $\mathrm{X}$ & - & $X$ \\
\hline Total & 209 & 60 & 28 \\
\hline
\end{tabular}


- UNESP/Campus de Jaboticabal, Departamento de Morfologia e Fisiologia Animal: Cynthia M. P. de A. Prado - ecologia e comportamento de anuros.

- UNESP/Campus de Rio Claro - Departamento de Zoologia: Augusto S. Abe, Célio F. B. Haddad, Denis Andrade - taxonomia, sistemática, biologia, comportamento, fisiologia e conservação de anfíbios.

- UNESP/Campus de Rio Claro, Departamento de Biologia: Sanae Kasahara - citogenética.

- UNESP/Campus de São José do Rio Preto, Departamento de Zoologia e Botânica: Denise de C. Rossa-Feres - ecologia de comunidades, ecologia, morfologia e taxonomia de girinos.

- UNESP/Campus de São José do Rio Preto, Departamento de Biologia: Classius de Oliveira - morfologia, biologia da reprodução e pigmentação visceral.

- UNICAMP/Campinas, Departamento de Biologia Celular: Shirlei Recco-Pimentel, Luciana Bolsoni Lourenço - citogenética animal e ultra-estrutura de espermatozóides, evolução, citotaxonomia e sistemática filogenética molecular.

- UNICAMP/Campinas, Museu de Zoologia "Adão José Cardoso": Ivan Sazima, Luis Felipe Toledo - taxonomia, história natural, comportamento, ecologia, eco-fisiologia e conservação de anfíbios.

- UNIFESP/Campus de Diadema, Departamento de Ciências Biológicas: Cinthia A. Brasileiro, João M. B. Alexandrino, José Eduardo de Carvalho, Ricardo J. Sawaya - taxonomia, sistemática, ecologia, biogeografia, genética, evolução, filogeografia, conservação e fisiologia de anfíbios.

- UNITAU/Taubaté, Departamento de Biologia: Itamar A. Martins - bioacústica, biologia, taxonomia e ecologia de anfíbios.

- USP- ESALQ/Piracicaba, Departamento de Ciências Biológicas: Jaime A. Bertoluci - ecologia, história natural, comportamento e conservação de anfíbios.

- USP/São Paulo, Departamento de Ecologia: Marcio R. C. Martins - ecologia de comunidades, ecologia comparativa e conservação.

- USP/São Paulo, Departamento de Fisiologia: Carlos Navas - fisiologia comparada, fisiologia evolutiva, ecofisiologia e biologia termal.

- USP/São Paulo, Departamento de Zoologia: Miguel T. U. Rodrigues - sistemática, zoogeografia e evolução.

- USP- Escola de Artes Ciências e Humanidades, Gestão Ambiental/São Paulo: Luis Schiesari - ecologia e ecotoxicologia de comunidades em sistemas aquáticos.

\section{Principais Lacunas do Conhecimento}

\section{Lacunas geográficas}

O mapa gerado pela análise de interpolação através dos registros de espécies presentes nas maiores Coleções do Estado de São Paulo (Figura 1) permitiu visualizar espacialmente duas grandes zonas com grande acúmulo de conhecimento sobre anfíbios anuros: principalmente as regiões das grandes cidades como São Paulo, Campinas e Santos, e também na região do Litoral Norte; e o noroeste do estado, principalmente nas imediações de São José do Rio Preto. Este grande acúmulo de dados se deve principalmente aos estudos desenvolvidos pelas universidades paulistas, mostrando a importância do estabelecimento de grandes centros de estudos em diferentes regiões do estado. Por outro lado, a alta concentração de estudos no litoral norte reflete principalmente o interesse de pesquisadores na grande biodiversidade de anfíbios encontrada nas unidades de conservação da Serra do Mar e litoral, sendo possível encontrar nestas localidades grande número de espécies endêmicas ou com registro único no estado e ameaçadas de extinção (e.g. Machado et al. 2005,
Sawaya \& Haddad 2006, São Paulo 2008, International Union for Conservation of Nature 2009).

Com relação às lacunas geográficas é evidente o desconhecimento da riqueza de anfíbios da região sudoeste do estado, principalmente na bacia hidrográfica do Rio Paranapanema. Além disso, é possível identificar também que existem lacunas na região nordeste, principalmente na divisa entre os Estados de Minas Gerais e São Paulo, embora a região tenha sido amostrada recentemente (Araujo et al. 2009). Esta região possui alta diversidade de paisagens, principalmente pelo fato de estar inserida em uma zona de transição dos biomas Mata Atlântica e Cerrado (Kronka et al. 2005). A região do Pontal do Paranapanema, apesar ter sido inventariada recentemente (Santos et al. 2009), ainda carece de informações, principalmente nas proximidades do Rio Paraná.

É importante ressaltar que informações sobre vários espécimes coletados no Estado por diversas instituições científicas não estão disponíveis publicamente, dificultando em muito o desenvolvimento científico e tecnológico de diversas áreas de conhecimento. A falta de informações sobre ocorrência de espécies, principalmente as endêmicas e ameaçadas, pode gerar informações imprecisas em projetos de conservação, como também em estudos de impactos ambientais. A Coleção de anfíbios do MZUSP é um exemplo claro desta problemática. Se os dados desta coleção fossem usados no presente trabalho possivelmente o resultado da interpolação do mapa seria diferente. Mas já foi iniciado um esforço para a finalização da digitação e conferência de registros desta base de dados (Projeto 11 Apêndice 1), que poderá estar disponível para análise em breve. A grande representatividade desta coleção, principalmente no Estado de São Paulo, poderia diminuir as áreas de lacunas e ressaltar um novo padrão do conhecimento dos anfíbios no estado. A dificuldade em obter essas informações contribui para o desconhecimento dos diversos padrões ecológicos das espécies, deixando de subsidiar políticas estaduais de conservação e de planejamento ambiental. Neste sentido, incentivar e apoiar a disponibilização dos dados das diversas coleções biológicas é de vital importância para o desenvolvimento sustentável e científico do estado.

\section{Lacunas de conhecimento}

A ausência de conhecimento dos processos envolvidos na origem e manutenção da biodiversidade de anfíbios no Brasil é uma lacuna importante a ser preenchida. A maior parte dos estudos em ecologia de anuros desenvolvidos no Estado de São Paulo, como no restante do país, são de cunho descritivo (veja abaixo Referências Bibliográficas; Apêndice 2: Referências Complementares sobre Anfíbios do Estado de São Paulo), o que é natural dada a grande diversidade de espécies e o desconhecimento de aspectos básicos de história natural, de uso de hábitat e de padrões de co-ocorrência e distribuição espacial e temporal (e.g., Bertoluci et al. 2007, Santos et al. 2007, Zina et al. 2007, Narvaes et al. 2009, Vasconcelos et al. 2009, Brassaloti et al. 2010). Estudos como esses, baseados em observações e amostragens na natureza, deverão continuar a ser desenvolvidos, pois só assim padrões naturais podem ser detectados e hipóteses a respeito de processos podem ser elaboradas. Entretanto, paralelamente a esses estudos, há evidente carência no conhecimento de processos que regulam populações e comunidades. Estes processos devem ser procurados em diversas escalas espaciais e temporais, integrando estudos que abordam a distribuição e abundância de espécies ou a composição e estrutura de comunidades com estudos que buscam entender como essas distribuições ou comunidades foram historicamente constituídas. A base de dados disponível atualmente permite que os estudos em ecologia avancem, empregando abordagens e métodos recentes, os quais englobam escalas menores e maiores do 


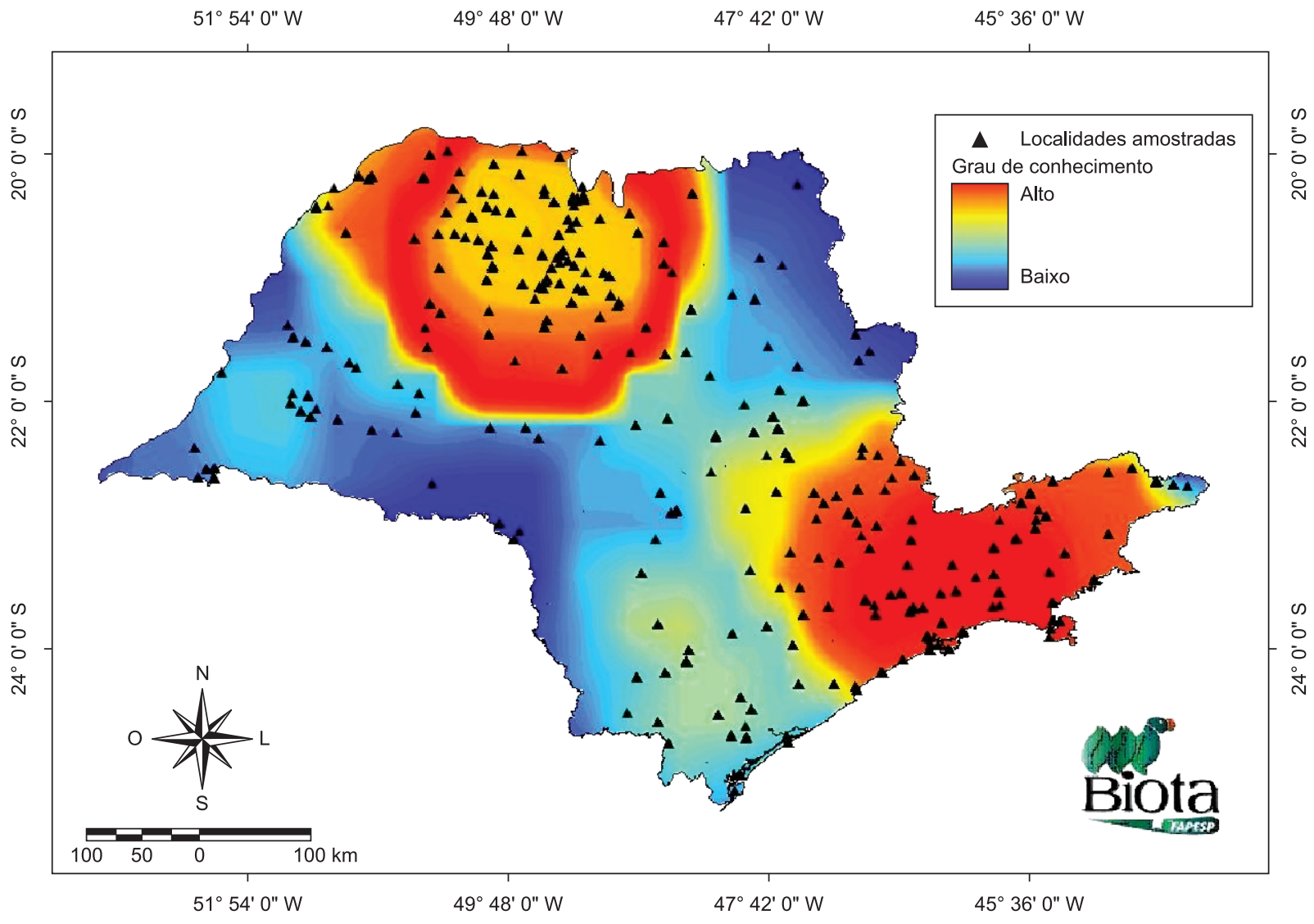

Figura 1. Mapa indicando a localização dos locais de amostragem de anfíbios anuros, com as áreas de maior conhecimento e as lacunas de amostragem ainda existentes no Estado de São Paulo.

Figure 1. Map showing the location of the sampling sites of anuran amphibians, with the most sampled areas and geographic sampling gaps still existing in the State of São Paulo.

que as espécies (e.g., Giovanelli et al. 2008a,b, 2010, Schiesari et al. 2009, Romero et al. 2010, Thomé et al. 2010).

Na escala abaixo da espécie, novas técnicas genéticas e moleculares desenvolvidas nas duas últimas décadas produziram e deverão continuar produzindo grande impacto na área de ecologia, evolução e conservação, introduzindo e aperfeiçoando temas como diversidade genética, resolução taxonômica, depressão endogâmica, fragmentação de populações, filogeografia, viabilidade genética de populações e reintrodução de espécies. Outra área promissora é a bioprospecção de princípios bioativos, haja vista a imensa quantidade de compostos já detectados na pele dos anfíbios (revisão em Schwartz et al. 2007).

$\mathrm{Na}$ escala acima da espécie, que engloba desde estudos experimentais em laboratório e no campo visando detectar o papel de processos bióticos (competição, predação) e abióticos na estrutura de populações e comunidades, estudos de modelagem de nicho ecológico que possibilitam predizer a ocorrência das espécies e de ecologia da paisagem, que considerem efeitos da estrutura e de alterações no hábitat e de poluição, até estudos de biogeografia e evolução, visando detectar a influência relativa de fatores históricos e contemporâneos na distribuição e regulação da diversidade de anuros, deverão ser conduzidos no contexto de metapopulações e metacomunidades, a fim de possibilitar testes de hipóteses em conservação.

Ao nível da espécie, estudos em dinâmica de populações, abordando flutuações populacionais em escalas temporais longas são urgentemente necessários para que se possa diferenciar declínios de flutuações populacionais naturais.
No contexto da história evolutiva das espécies, estudos filogeográficos (sensu Avise 2000) permitirão elucidar a dinâmica histórica da diversificação biológica, tanto dentro de espécies como em grupos de espécies filogeneticamente próximas. Os poucos estudos publicados até o presente que incluíram amostragem no Estado de São Paulo mostraram a utilidade da filogeografia para testar hipóteses sobre a biogeografia histórica da anurofauna da Mata Atlântica, discutindo especialmente se refúgios pleistocênicos de mata ou barreiras fluviais teriam atuado na origem da diversidade biológica observada nesse bioma (Carnaval et al. 2009, Fitzpatrick et al. 2009, Thomé et al. 2010). Por exemplo, esses estudos revelaram pelo menos uma grande fronteira biogeográfica na Mata Atlântica do estado, a sudoeste da cidade de São Paulo, separando linhagens evolutivas e/ou espécies que estiveram isoladas historicamente por longos períodos de tempo. No futuro, espera-se um aumento pronunciado de estudos filogeográficos em anfíbios, permitindo análises comparativas de espécies e grupos de espécies com histórias de vida diversas (e.g., modos reprodutivos). Este conhecimento permitirá colocar numa perspectiva histórica as origens da diversidade e abundância de espécies, tanto de áreas do Estado de São Paulo como dos biomas que aí se encontram.

\section{Perspectivas para os Próximos 10 Anos}

\section{Taxonomia e sistemática}

Embora o Estado de São Paulo seja a região melhor estudada no Brasil, há ainda áreas que correspondem a lacunas de conhecimento 
como demonstrado neste estudo (Figura 1). Certamente serão encontradas espécies novas, ainda não descritas, bem como novos registros à medida que inventários em áreas não estudadas ou pouco estudadas se intensifiquem.

Aliado aos estudos em taxonomia está o conhecimento das linhagens evolutivas das espécies. Os estudos sobre relações filogenéticas não obedecem às fronteiras políticas e, portanto, a discussão sobre a sistemática dos anfíbios do Estado de São Paulo necessariamente deve ocorrer no contexto de todos os clados que contenham espécies distribuídas nos biomas da Mata Atlântica e Cerrado, no Sudeste do Brasil. Embora ainda bastante incipiente, o conhecimento acerca das relações filogenéticas de clados de anuros com espécies presentes nesta área tem experimentado um crescimento constante nos últimos cinco anos. Os estudos que embasam este crescimento incluem análises de clados ao nível de espécie com algumas poucas espécies desta área (e.g., Mendelson III et al. 2000, Trueb \& Massemin 2001, Chek et al. 2001, Grant et al. 2006, Aguiar Junior. et al. 2007, Garda \& Cannatella 2007, Wiens et al. 2007, Guayasamin et al. 2008, Ponssa 2008, Wiens et al. 2008), clados nos quais as espécies do sudeste do Brasil predominam ou representam uma parte importante da diversidade tratada (Faivovich et al. 2004, 2010, Lourenço et al. 2008, Thomé et al. 2010), ou estudos de grandes grupos nos quais os anfíbios do Sudeste do Brasil representaram parte da diversidade filogenética, mas sem o objetivo de se ater à abordagem ao nível de espécie para a maioria deles (Faivovich 2002, Haas 2003, Darst \& Cannatella 2004, Faivovich et al. 2005, Frost et al. 2006, Pramuk 2006, Pramuk et al. 2007, van der Meijden et al. 2007, Hedges et al. 2008, Heinicke et al. 2009, van Bocxlaer et al. 2010, Wiens et al. 2006: supp data, Wiens et al. 2010). Os benefícios resultantes destes estudos para o conhecimento dos anuros do sudeste brasileiro variam desde a produção de filogenias com comentários extensivos (e.g., Faivovich 2002, Faivovich et al. 2005, 2010, Hedges et al. 2008, Guayasamin et al. 2008, Ponssa 2008, Lourenço et al. 2007, Thomé et al. 2010) até árvores filogenéticas sem nenhum comentário em particular sobre as espécies ou grupos de espécies desta região do Brasil (Wiens et al. 2006:supp data).

Os próximos 10 anos testemunharão um florescimento sem precedentes de estudos filogenéticos de clados de anuros endêmicos ao sudeste do Brasil ou com espécies presentes nesta área. Esta tendência é evidente considerando-se o grande número de pesquisadores, a maioria brasileiros ou pesquisadores estrangeiros trabalhando em instituições brasileiras, que estudam as relações filogenéticas de diversos grupos. O que podemos esperar em termos de resultados destes estudos? Primeiramente, podemos esperar hipóteses filogenéticas ao nível específico para a maioria dos clados relevantes. Em segundo lugar, seria particularmente importante ter hipóteses filogenéticas bem suportadas entre as famílias de anuros correntemente reconhecidas, particularmente as de Hyloides, cujas hipóteses filogenéticas atuais estão ainda pouco suportadas. Se este cenário irá se concretizar ou não, dependerá de vários fatores, como por exemplo, se a sistemática irá se beneficiar dos dados que deverão ser disponibilizados pela revolução genômica em curso. Em terceiro lugar, e agora levando em conta o que gostaríamos de compreender, nós estaríamos próximos do entendimento de um assunto que está emergindo aos poucos dos resultados filogenéticos e que tem sido comentado apenas raramente: Qual foi o papel histórico do que hoje é o Sudeste do Brasil na diversificação dos Hyloides?

É importante pensarmos na qualidade da sistemática que desejamos desenvolver. Uma boa sistemática é feita, por definição, por bons sistematas e taxonomistas, e não por técnicos que realizam sequenciamento ou por estagiários de cursos da graduação. Os bons sistematas e taxonomistas são os que dispõem do conhecimento de primeira mão acerca dos animais estudados. Isto deriva, em grande parte, não do conhecimento gestáltico que um pesquisador obtenha no campo, pois frequentemente este tipo de conhecimento não é palpável para o estabelecimento de hipóteses de relações filogenéticas, mas sim, e de forma mais relevante, dos estudos da morfologia dos anuros feitos sob o estereomicroscópio.

A proposição de hipóteses filogenéticas com o uso integrado de dados fenotípicos, advindos da morfologia e da biologia das espécies, idealmente analisados simultaneamente com os dados moleculares, mas também embasando discussões minimamente adequadas, provavelmente será o fator que diferenciará uma fábrica de produção em massa de filogenias de uma disciplina científica que cresce em bases sólidas. Além disso, esta integração será o instrumental para aumentar o conhecimento taxonômico dos anuros, pois as sinapomorfias fenotípicas ainda são desconhecidas para a maioria dos gêneros e agrupamentos supra-específicos. Nesta integração será chave a inclusão de informações sobre fósseis, que por sua vez possibilitarão uma melhor calibração das estimativas de tempo de divergência, permitindo que cada vez usemos menos, ou idealmente não usemos mais, as evidências indiretas e muitas vezes dúbias, como aquelas baseadas nos eventos tectônicos ou nas taxas evolutivas fixas para genes específicos.

O futuro do nosso conhecimento filogenético dos clados de anuros endêmicos ou que contenham espécies do sudeste do Brasil parece muito promissor. É evidente que o desafio mais importante será o uso criativo e inteligente que faremos deste conhecimento.

\section{Ecologia}

Em uma escala regional, é aparente o progresso no conhecimento de padrões de distribuição e diversidade de anfíbios do Estado de São Paulo nos últimos anos, e de forma clara a contribuição do Programa BIOTA/FAPESP. Este conhecimento permitiu o desenvolvimento dos primeiros estudos biogeográficos com implicações para a definição de áreas prioritárias para a conservação de anfíbios no estado (e.g., Rossa-Feres et al. 2008, Becker et al. 2010b).

Em uma escala mais local, o estudo de assembléias de anfíbios, ou comunidades que incluem anfíbios, em raras ocasiões estendeuse além da detecção de padrões de abundância espacial e temporal. Em outras palavras, se por um lado avançamos no conhecimento da estrutura de determinadas comunidades, ainda é bastante incipiente o conhecimento dos processos ecológicos que as organizam. Dentro deste contexto, é importante realçar o amplo predomínio de estudos de natureza observacional e, consequentemente, a raridade de estudos de natureza experimental, no estudo de anfíbios em São Paulo e no Brasil. Tal raridade chama a atenção, especialmente se considerarmos que girinos constituem um importante sistema-modelo em ecologia experimental (Wilbur 1997) e contribuíram com importantes avanços no nosso conhecimento de questões conceituais e gerais a respeito da forma como a natureza é estruturada (e.g., Wilbur 1976, 1987, Morin 1983, Werner 1992, 1998, Wellborn et al. 1996, Werner \& Peacor 2003). Esta falta de tradição no emprego de anfíbios como sistema experimental indica um relativo atraso na ecologia experimental brasileira como um todo e é esperado que nos próximos anos haja um avanço mais efetivo neste campo. Para isso, a abordagem experimental deverá ser incentivada pelas agências de fomento, de modo a superar uma tradição no desenvolvimento de estudos baseados em observação. Apesar da abordagem experimental não ser o único caminho para avançarmos em direção a uma ecologia preditiva, sua importância reside em esclarecer os mecanismos de processos ecológicos em várias escalas como, por exemplo, seleção de hábitat, montagem de comunidades e efeitos de alterações ambientais e poluição (e.g., Resetaritis Junior \& Bernardo 1998, Wilbur 1997, Schiesari et al. 2009), os quais interagem de modo tão complexo que é quase impossível avaliar seu efeito por meio de observações e correlações (Wilbur 1997, Werner 1998). 
Além disso, embora tenha sido notável o avanço no conhecimento da história natural dos anfíbios paulistas nos últimos anos (e.g., Becker et al. 2010a,b), permanece uma carência considerável em estudos de dinâmica de populações. Esta carência se manifesta em estudos demográficos de curta duração que permitem a construção de tabelas de vida a partir da quantificação empírica de taxas de sobrevivências e fecundidades associadas a cada sexo e estágio e/ou idade. Também se manifesta em estudos demográficos de longa duração que permitam uma avaliação quantitativa das flutuações naturais de populações, bem como da ocorrência de eventos naturais de extinção e colonização (e.g., Bertoluci \& Heyer 1995), que são na verdade comuns e próprios da dinâmica de metapopulações de anfíbios em outros sistemas (Skelly et al. 2003). Seria interessante que estes estudos de longa duração focassem em localidades já bem conhecidas para expandir sobre dados históricos de presença/ausência ou mesmo tamanhos populacionais de espécies, inclusive nas duas localidades do Estado reconhecidas como tendo sido passíveis de declínios de populações de anfíbios, como a Estação Biológica de Boracéia e a Reserva Biológica de Paranapiacaba. Uma terceira localidade hipotetizada como abrigando espécies passíveis de declínio a partir de informações mais anedóticas é Campos do Jordão (Verdade et al. no prelo). É surpreendente a falta de estudos, nestas e em outras localidades, direcionados a testar de fato evidências de declínio de populações, ou a presença atual de agentes capazes de terem levado a declínios, como a incidência de doenças e a presença de poluentes.

No que diz respeito a mecanismos de perda de espécies, recebeu boa atenção recente a desconexão de hábitat ou habitat split (Becker et al. 2007, 2009), uma nova hipótese testada e confirmada para espécies de anuros através de projetos financiados pelo Programa Biota/FAPESP. A desconexão de hábitat afeta as espécies com ciclo de vida bifásico, resultando em grande declínio populacional e extinções após poucas gerações (Becker et al. 2007, 2009). São anedóticos os estudos de impactos decorrentes da introdução de espécies exóticas (mas veja Giovanelli et al. 2008b), incidência de doenças (Carnaval et al. 2006, Toledo et al. 2006a,b) e poluentes. Com relação à ecotoxicologia, uma revisão recente mostrou que a absoluta maioria dos estudos de efeitos de poluentes sobre anfíbios focou espécies comuns e amplamente distribuídas do hemisfério norte, e até recentemente não havia estudos quantificando efeitos de qualquer poluente sobre anfíbios brasileiros (Schiesari et al. 2007; mas veja, por exemplo, Leite et al. 2010). É mais um campo onde se espera que haja grande progresso nos próximos anos, mesmo porque importantes avanços na frutífera aproximação recente da ecologia com a ecotoxicologia estão se originando de pesquisas com anfíbios (e.g., Relyea et al. 2005).

Em função das linhas de pesquisa recentemente implantadas no Estado de São Paulo, outras duas abordagens deverão apresentar grande crescimento nos próximos 10 anos: estudos empregando técnicas de modelagem espacial (e.g., Giovanelli et al. 2008a, b, 2010, Vasconcelos et al. 2010) e estudos de filogeografia (e.g., Carnaval et al. 2009, Fitzpatrick et al. 2009, Thomé et al. 2010). Essas duas abordagens, especialmente se associadas, permitirão uma abordagem evolutiva no estudo da distribuição espacial de espécies e comunidades, buscando detectar os processos históricos que as constituíram.

\section{Conservação: integrando ecologia e sistemática}

Apesar dos animais não se distribuírem de forma a obedecer fronteiras políticas, e certamente ser necessário analisar a distribuição completa de todas as espécies de anfíbios que ocorrem no Estado de São Paulo, precisamos considerar que cada estado possui suas políticas próprias de conservação e meios para executá-las. Assim, cada estado também deve considerar prioritária para conservação uma espécie que seja, por exemplo, de distribuição restrita no Estado, mas ampla em outras regiões, de forma a garantir a permanência e conservação dessa espécie, caso o mesmo não seja realizado nos estados vizinhos.

No Brasil, a definição de espécies ameaçadas de extinção ou espécies-alvo para conservação tem sido baseada em critérios internacionais (cf. Internacional... 2001) que incluem: os tipos de ameaças sofridas por determinada espécie; a proporção de declínio populacional em um intervalo de tempo, suas causas, incluindo destruição ou degradação de hábitats, espécies exóticas invasoras, patógenos e poluição; a extensão da distribuição geográfica; a área de ocorrência; e o tamanho da população. Em avaliação recente das espécies de anfíbios ameaçadas do Estado de São Paulo (Garcia et al. 2009), foram analisadas 225 espécies, das quais apenas 11 foram consideradas ameaçadas de extinção (cerca de 5\% do total) e uma extinta. Ainda nesta avaliação, sete espécies foram incluídas na categoria Quase Ameaçada (3\%), pela falta de informação sobre seu status populacional, e 45 espécies (20\%) foram consideradas como sem informação suficiente para serem avaliadas, sendo incluídas na categoria Dados Deficientes. Apesar de relativamente objetivos e amplamente utilizados, esses critérios para avaliação de espécies ameaçadas são baseados em informações detalhadas sobre parâmetros populacionais, distribuição das espécies e degradação ambiental, dados estes não disponíveis para grande parte das espécies e localidades.

As poucas espécies diagnosticadas como ameaçadas de extinção certamente devem receber atenção especial. Mas algo que ainda não tem sido considerado no Brasil como critério para definição de espécies prioritárias para conservação, além do risco de extinção, é a conservação da diversidade filogenética. A importância de conservar grupos filogenéticos distintos tem sido enfatizada nos últimos anos (e.g. Vázquez \& Gittleman 1998, Bininda-Emonds et al. 2000, Mace et al. 2003, Purvis et al. 2005). Considerar a diversidade de linhagens filogenéticas permite preservar além das espécies, a história evolutiva de determinada região ou biota, pois os resultados dos processos ecológicos e evolutivos estarão representados nas espécies das diferentes linhagens de uma determinada área (Mace et al. 2003). A partir dos rápidos avanços que devem ocorrer na próxima década em relação à compreensão das relações de parentesco dos anfíbios (ver item Perspectivas para os Próximos 10 anos - Taxonomia e Sistemática, neste estudo) será possível implementar a conservação da biodiversidade filogenética aos critérios usualmente utilizados para a definição de espécies prioritárias para conservação e pesquisa.

\section{Agradecimentos}

Agradecemos Victor D. Orrico, Bianca Berneck, Amon Mendes-Luiz, Thiago A. Pires e Sérgio Serrano-Filho pela ajuda com a bibliografia. Célio F. B. Haddad, Denise de C. Rossa-Feres, João Alexandrino, Luis Schiesari e Ricardo J. Sawaya agradecem à FAPESP (respectivamente Procs. 01/13341-3 e 08/50928-1; 04/04820-3; 05/52727-5; 08/57939-9; 08/54472-2). Ricardo J. Sawaya agradece ao INCTTOX. Julián Faivovich agradece a ANPCyT PICT 223-2006 e 2202-2007. Agradecemos a Hebert Ferrarezzi pelas sugestões. C.F.B. Haddad, D.C. Rossa-Feres e R.J. Sawaya são bolsistas do CNPq.

\section{Referências Bibliográficas}

AGUIAR-JUNIOR, O., BACCI JUNIOR, M., LIMA, A.P., ROSSA-FERES, D.C., HADDAD, C.F.B. \& RECCO-PIMENTEL, S.M. 2007. Phylogenetic relationships of Pseudis and Lysapsus (Anura, Hylidae, Hylinae) inferred from mitochondrial and nuclear gene sequences. Cladistics 23:455-463. http://dx.doi.org/10.1111/j.1096-0031.2007.00154.x 
ARAUJO, C.O., CONDEZ, T.H. \& HADDAD, C.F.B. 2007a. Amphibia Anura, Barycholos ternetzi, Chaunus rubescens, and Scinax canastrensis: distribution extension, new state record. Check List 3:153-155.

ARAUJO, C.O., CONDEZ, T.H. \& HADDAD, C.F.B. 2007b. Amphibia, Anura, Phyllomedusa ayeaye (B. Lutz, 1966): distribution extension, new state record, and geographic distribution map. Check List 3:156-158.

ARAUJO, C.O., CONDEZ, T.H. \& SAWAYA, R.J. 2009. Anfíbios anuros do Parque Estadual das Furnas do Bom Jesus, sudeste do Brasil, e suas relações com outras taxocenoses no Brasil. Biota Neotrop. 9(2):1-22: http://www. biotaneotropica.org.br/v9n2/pt/abstract?article+bn01309022009 (último acesso em 18/06/2010).

ARAÚJO, O.G.S., TOLEDO, L.F., GARCIA, P.C.A. \& HADDAD, C.F.B 2009. The amphibians of São Paulo State, Brazil. Biota Neotrop 2009, 9(4): 197-209: http://www.biotaneotropica.org.br/v9n4/pt/abstract?inve ntory+bn03109042009 (último acesso em 19/07/2010).

AVISE, J.C. 2000. Phylogeography: the history and formation of species. Harvard University Press, Cambridge.

BECKER, C.G., FONSECA, C.R., HADDAD, C.F.B., BATISTA, R.F. \& PRADO, P.I. 2007. Habitat split and the global decline of amphibians. Science 318:1775-1777. http://dx.doi.org/10.1126/science.1149374

BECKER, C.G., FONSECA, C.R., HADDAD, C.F.B. \& PRADO, P.I. 2009 Habitat split as a cause of local population declines of amphibians with aquatic larvae. Cons. Biol. 24(1): 287-294. http://dx.doi.org/10.1111/ j.1523-1739.2009.01324.x

BECKER, C.G., LOYOLA, R.D., HADDAD, C.F.B. \& ZAMUDIO, K.R. 2010b. Integrating species life-history traits and patterns of deforestation in amphibian conservation planning. Divers. Distrib. 16:10-19. http:// dx.doi.org/10.1111/j.1472-4642.2009.00625.x

BERNECK, B.V.M., COSTA, C.O.R. \& GARCIA, P.C.A. 2008. A new species of Leptodactylus (Anura: Leptodactylidae) from the Atlantic Forest of São Paulo State, Brazil. Zootaxa 1795:46-56.

BERTOLUCI, J. \& HEYER, W.R. 1995. Boracéia update. Froglog 14:2-3.

BERTOLUCI, J., BRASSALOTI, R.A., RIBEIRO JÚNIOR, J.W., VILELA, V.M.F.N. \& SAWAKUCHI, H.O. 2007. Species composition and similarities among anuran assemblages of forest sites in southeastern Brazil. Sci. Agric 64:364-374. http://dx.doi.org/10.1590/S010390162007000400007

BININDA-EMONDS, O.R.P., VÁZQUEZ, D.P. \& MANNE, L.L. 2000. The calculus of biodiversity: integrating phylogeny and conservation. Trends Ecol. \& Evol.15:92-94. http://dx.doi.org/10.1016/S0169-5347(99)017814

BLAUSTEIN, A.R. \& WAKE, D.B. 1990. Declining amphibian populations: a global phenomenon? Trends Ecol. \& Evol. 5:203-204.

BOKERMANN, W.C.A. 1966. A new Phyllomedusa from Southeastern Brazil. Herpetologica 22(4):293-298.

BOSCH, J. 2003. Nuevas amenazas para los anfibios: enfermedades emergentes. Munibe (16):56-73. Supl.

BRASILEIRO, C.A., HADDAD, C.F.B., SAWAYA, R.J. \& MARTINS, M. 2007a. A new and threatened species of Scinax (Anura; Hylidae) from Queimada Grande Island, southeastern Brazil. Zootaxa 1391:47-55.

BRASILEIRO, C.A., HADDAD, C.F.B., SAWAYA, R.J. \& SAZIMA, I. 2007b. A new and threatened island-dwelling species of Cycloramphus (Anura: Cycloramphidae) from southeastern Brazil. Herpetologica 32:501-510.

BRASILEIRO, C.A., OYAMAGUCHI, H.M. \& HADDAD, C.F.B. 2007c. A new island species of Scinax (Anura; Hylidae) from southeastern Brazil. J. Herpetol. 41:271-275.

BRASSALOTI, R.A., ROSSA-FERES, D.C. \& BERTOLUCI, J. 2010. Anurofauna da floresta estacional semidecidual da Estação Ecológica dos Caetetus, sudeste do Brasil. Biota Neopropica 10:1-17: http://www. biotaneotropica.org.br/v10n1/pt/abstract?article+bn01810012010 ISSN 1676-0603 (último acesso em 18/06/2010).

CARNAVAL, A.C.O.Q., HICKERSON, M.J., HADDAD, C.F.B., RODRIGUES, M.T. \& MORITZ, C. 2009. Stability predicts genetic diversity in the Brazilian Atlantic Forest hotspot. Science 323:785-789. http://dx.doi.org/10.1126/science.1166955
CARNAVAL, A.C.O.Q., PUSCHENDORF, R., PEIXOTO, O.L., VERDADE, V.K. \& RODRIGUES, M.T. 2006. Amphibian chytrid fungus broadly distributed in the Brazilian Atlantic Rain Forest. EcoHealth 3:41-48. http://dx.doi.org/10.1007/s10393-005-0008-2

CHEK, A.A., LOUGHEED, S.C., BOGART, J.P. \& BOAG, P.T. 2001. Perception and history: molecular phylogeny of a diverse group of Neotropical frogs, the 30-chromosome Hyla (Anura: Hylidae). Mol. Phylogenet. Evol. 18:370-385. http://dx.doi.org/10.1006/mpev.2000.0889

CRESSIE, N.A.C. 1991. Statistics for spatial data. John Wiley \& Sons, New York, 920p.

COCHRAN, D.M. 1938. Diagnoses of new frogs from Brazil. Proceedings of the Biological Society of Washington 51:41-42.

DARST, C.R. \& CANNATELLA, D.C. 2004. Novel relationships among hyloid frogs inferred from $12 \mathrm{~S}$ and $16 \mathrm{~S}$ mitochondrial DNA sequences. Mol. Phylogenet. Evol. 31:462-475. http://dx.doi.org/10.1016/j. ympev.2003.09.003

DUELLMAN, W.E. 1988. Pattern of species diversity in anuran amphibians in the American tropics. Ann. MO Bot. Gard. 75:79-104. http://dx.doi. org/10.2307/2399467

DUELLMAN, W.E. \& TRUEB, L. 1994. Biology of amphibians. McGrawHill, Baltimore; London.

FAIVOVICH, J. 2002. A cladistic analysis of Scinax (Anura: Hylidae). Cladistics 18:367-393. http://dx.doi.org/10.1111/j.1096-0031.2002. tb00157.x

FAIVOVICH, J., GARCIA, P.C.A., ANANIAS, F., LANARI, L., BASSO, N.G. \& WHEELER, W.C. 2004. A molecular perspective on the phylogeny of the Hyla pulchella species group (Anura, Hylidae). Molecular Phylogenetics and Evolution 32:938-950. http://dx.doi.org/10.1016/j. ympev.2004.03.008

FAIVOVICH, J., HADDAD, C.F.B., GARCIA, P.C.A., FROST, D.R., CAMPBELL, J.A. \& WHEELER, W.C. 2005. Systematic review of the frog family Hylidae, with special reference to Hylinae: phylogenetic analysis and taxonomic revision. Bulletin of the American Museum of Natural History 294:1-240. http://dx.doi.org/10.1206/00030090(2005)294[0001:SROTFF]2.0.CO;2

FAIVOVICH, J., HADDAD, C.F.B., BAÊTA, D., JUNGFER, K.H., ÁLVARES, G.F.R., BRANDÃO, R.A., SHEIL, C., BARRIENTOS, L.S., BARRIO-AMRÓS, C.L., CRUZ, C.A.G. \& WHEELER, W.C. 2010. The phylogenetic relationships of the charismatic poster frogs, Phyllomedusinae (Anura, Hylidae). Cladistics 26:227-261. http://dx.doi org/10.1111/j.1096-0031.2009.00287.x

FITZPATRICK, S.W., BRASILEIRO, C.A., HADDAD, C.F.B. \& ZAMUDIO, K.R. 2009. Geographical variation in genetic structure of an Atlantic Coastal Forest frog reveals regional differences in habitat stability. Molecular Ecology 18:2877-2896. http://dx.doi.org/10.1111/j.1365294X.2009.04245.x

FROST, D.R., GRANT, T., FAIVOVICH, J., BAIN, R., HAAS, A., HADDAD, C.F.B., DE SÁ, R.O., CHANNING, A., WILKINSON, M., DONNELLAN, S.C., RAXWORTHY, C.J., CAMPBELL, J.A., BLOTTO, B.L., MOLER, P., DREWES, R.C., NUSSBAUM, R.A., LYNCH, J.D., GREEN, D. \& WHEELER, W.C. 2006. The amphibian tree of life. Bull. Am. Mus. Nat. Hist. 297:1-370. http://dx.doi. org/10.1206/0003-0090(2006)297[0001:TATOL]2.0.CO;2

FROST, D.R. 2010. Amphibian species of the world: an online reference. Version 5.4 (8 April, 2010). Electronic Database accessible at http:// research.amnh.org/vz/herpetology/amphibia (último acesso em 28/06/2010). American Museum of Natural History, New York.

GARCIA, P.C.A., SAWAYA, R.J., MARTINS, I.A., BRASILEIRO, C.A., VERDADE, V.K., JIM, J., SEGALLA, M.V., MARTINS, M., ROSSAFERES, D.C., HADDAD, C.F.B., TOLEDO, L.F., PRADO, C.P.A., BERNECK, B.M. \& ARAÚJO, O.G.S. 2009. Anfíbios. In Fauna ameaçada de extinção no Estado de São Paulo, Vertebrados (P.M. Bressan, M.C.M. Kierulff \& A.M. Sugieda, eds.). Governo do Estado de São Paulo, Secretaria do Meio Ambiente, Fundação Parque Zoológico de São Paulo, São Paulo, p. 329-347. 
GARDA, A.A. \& CANNATELLA, D.C. 2007, Phylogeny and biogeography of paradoxical frogs (Anura, Hylidae, Pseudae) inferred from 12S and 16S mitochondrial DNA. Mol. Phylogenet. Evol. 44:104-114. http://dx.doi. org/10.1016/j.ympev.2006.11.028

GIOVANELLI, J.G.R., ARAUJO, C.O., HADDAD, C.F.B., ALEXANDRINO, J. 2008a. Modelagem do nicho ecológico de Phyllomedusa ayeaye (Anura: Hylidae): previsão de novas áreas de ocorrência para uma espécie rara. Neotrop. Biol. Cons. 3:59-65.

GIOVANELLI, J.G.R., HADDAD, C.F.B., ALEXANDRINO, J. 2008b. Predicting the potential distribution of the alien invasive American bullfrog (Lithobates catesbeianus) in Brazil. Biol. Invasions 10:585-590. http://dx.doi.org/10.1007/s10530-007-9154-5

GIOVANELLI, J.G.R., DE SIQUEIRA, M.F., HADDAD, C.F.B. \& ALEXANDRINO, J. 2010. Modeling a spatially restricted distribution in the Neotropics: how the size of calibration area affects the performance of five presence-only methods. Ecol. Model. 221:215-224. http://dx.doi. org/10.1016/j.ecolmodel.2009.10.009

GRANT, T., FROST, D.R., CALDWELL, J.P., GAGLIARDO, R., HADDAD, C.F.B., KOK, P.J.R., MEANS, B.D., NOONAN, B.P., SCHARGEL, W.E. \& WHEELER, W.C. 2006 Phylogenetic systematics of dart-poison frogs and their relatives (Amphibia: Athesphatanura: Dendrobatidae). Bull. Am. Mus. Nat. Hist. 299:1-262. http://dx.doi.org/10.1206/00030090(2006)299[1:PSODFA]2.0.CO;2

GUAYASAMIN, J.M., CASTROVIEJO-FISHER, S., TRUEB, L., AYARZAGÚENA, J., RADA, M. \& VILÁ, C. 2008. Phylogenetic systematics of glassfrogs (Amphibia: Centrolenidae) and their sister taxon Allophryne ruthveni. Zootaxa 2100:1-97.

HAAS, A. 2003. Phylogeny of frogs as inferred from primarily larval characters (Amphibia: Anura). Cladistics 19:23-89.

HADDAD, C.F.B. 1998. Biodiversidade dos anfíbios no Estado de São Paulo. In Biodiversidade do Estado de São Paulo, Brasil: síntese do conhecimento ao final do século XX. Vertebrados (R.M.C. Castro, C.A. Joly.\& C.E.M. Bicudo, eds.). WinnerGraph, São Paulo, v.6, p.15-26.

HADDAD, C.F.B. \& PRADO, C.P.A. 2005. Reproductive modes in frogs and their unexpected diversity in the Atlantic Forest of Brazil. BioScience 55:207-217. http://dx.doi.org/10.1641/0006-3568(2005)055[0207:RM IFAT]2.0.CO;2

HEDGES, S.B., DUELLMAN, W.E. \& HEINICKE, M.P. 2008. New world direct-developing frogs (Anura: Terrarana): Molecular phylogeny, classification, biogeography, and conservation. Zootaxa 1737:1-182.

HEINICKE, M.P., DUELLMAN, W.E., TRUEB, L., MEANS, D.B., MACCULLOCH, R.D. \& HEDGES, S.B. 2009. A new frog family (Anura: Terrarana) from South America and an expanded directdeveloping clade revealed by molecular phylogeny. Zootaxa: 2211:1-35.

HIJMANS, R.J., GUARINO, L. \& ROJAS, E. 2002. DIVA-GIS. A geographic information system for the analysis of biodiversity data. Manual. International Potato Center, Lima, 73p.

HÖDL, W. 1990. Reproductive diversity in Amazonian lowland frogs. Fortschr. Zool. 38:41-60.

INTERNATIONAL UNION FOR CONSERVATION OF NATURE - IUCN. 2009. Dispõe de informações sobre a lista internacional das espécies ameaçadas (Red List of Threatened Species). Versão 2009. Disponível em: http://www.iucnredlist.org (último acesso em 11/06/2010).

INTERNATIONAL UNION FOR CONSERVATION OF NATURE - IUCN. 2001. IUCN red list categories and criteria: Version 3.1. IUCN Species Survival Comission. IUCN, Gland, Switzerland; Cambridge, UK, 30p.

JOLY, C.A., RODRIGUES, R.R., METZGER, J.P., HADDAD, C.F.B., VERDADE, L.M., OLIVEIRA, M.C. \& BOLZANI, V.S. 2010. Biodiversity conservation research, training, and policy in São Paulo. Science 328:1358-1359. http://dx.doi.org/10.1126/science.1188639

KIESECKER, J.M., BLAUSTEIN, A.R. \& BELDEN, L.K. 2001. Complex causes of amphibian population declines. Nature 410:681-684.
KRONKA, F.J.N., NALON, M.A., MATSUKUMA, C.K., KANASHIRO, M.M., YWANE, M.S.S., PAVÃO, M., DURIGAN, G., LIMA, L.P.R., GUILLAUMON, J.R., BAITELLO, J.B., BORGO, S.C., MANETTI, L.A., BARRADAS, A.M.F, FUKUDA, J.C., SHIDA, C.N., MONTEIRO, C.H.B., PONTINHAS, A.A.S., ANDRADE, G.G., BARBOSA, O., SOARES, A.P., JOLY, C.A. \& COUTO, H.T.Z. 2005. Inventário florestal da vegetação nativa do Estado de São Paulo. Secretaria do Meio Ambiente, Instituto Florestal, 200p.

LEITE, P.Z., MARGARIDO, T.C.S., LIMA, D., ROSSA-FERES, D.C. \& ALMEIDA, E.A. 2010. Esterase inhibition in tadpoles of Scinax fuscovarius (Anura, Hylidae) as biomarker of exposure to organophosphate pesticides. Environ. Sci. Pollut. Res. Int. 17:1411-1421. PMid:20383595. http://dx.doi.org/10.1007/s11356-010-0326-y

LIPS, K.R. 1999. Mass mortality and population declines of anurans at an upland site in western Panamá. Cons. Biol. 13:117-125. http://dx.doi. org/10.1046/j.1523-1739.1999.97185.x

LOURENÇO, L.B., BACCI JUNIOR, M., MARTINS, V.G., RECCOPIMENTEL, S.M. \& HADDAD, C.F.B. 2007. Molecular phylogeny and karyotype differentiation in Paratelmatobius and Scythrophrys (Anura, Leptodactylidae). Genetica 132:255-266. http://dx.doi.org/10.1007/ s10709-007-9169-y

LUTZ, A. 1925a. Batracien du Brésil. Comptes rendus Soc. Biol. Paris 93(1):137-139.

LUTZ, A. 1925b. Batracien du Brésil (II). Comptes rendus Soc. Biol. Paris 93(22):211-224.

LUTZ, A. 1929. Taxonomia e biologia do gênero Cyclorhampus. Memórias do Instituto Oswaldo Cruz 22(1):3-23.

MACE, G.M., GITTLEMAN, J.L. \& PURVIS, A. 2003. Preserving the tree of life. Science 300:1707-1709. http://dx.doi.org/10.1126/science. 1085510

MACHADO, A.B.M., MARTINS, C.S. \& DRUMMOND, G.M. 2005. Lista das espécies da fauna brasileira ameaçadas de extinção. Fundação Biodiversitas, Belo Horizonte, 160p.

McDIARMID, R.W. 1994. Amphibian diversity and natural history: an overview (W.R. Heyer, M.A. Donnelly, R.W. McDiarmid, L.A.C. Hayek \& M.S. Foster, eds.). Measuring and monitoring biological diversity: standard methods for amphibians. Smithsonian Institution Press, Washington, p.5-15.

MENDELSON III, J.R., DA SILVA, H.R. \& MAGLIA, A.M. 2000. Phylogenetic relationships among marsupial frog genera (Anura: Hylidae: Hemiphractinae) based on evidence from morphology and natural history. Zool. J. Linn. Soc. London 128:125-148. http://dx.doi. org/10.1111/j.1096-3642.2000.tb00159.x

METZGER, J.P. \& CASATTI, L. 2006. Do diagnóstico à conservação da biodiversidade: o estado da arte do programa BIOTA/FAPESP. Biota Neotrop. 6(2): http://www.biotaneotropica.org.br/v6n2/pt/abstract?pointof-view+bn00106022006 (último acesso em 18/06/2010).

MORIN, P.J. 1983. Predation, competition, and the composition of larval anuran guilds. Ecol. Monogr. 53(2):119-138. http://dx.doi. org/10.2307/1942491

NARVAES, P., BERTOLUCI, J. \& RODRIGUES, M.T. 2009. Composição, uso de hábitat e estações reprodutivas das espécies de anuros da floresta de restinga da Estação Ecológica Juréia-Itatins, sudeste do Brasil. Biota Neotrop. 9:117-123: http://www.biotaneotropica.org.br/v9n2/pt/ abstract?article+bn02009022009 ISSN 1676-0603 (último acesso em 18/06/2010).

PECHMAN, J.H.K., SCOTT, D.E., SEMLITSCH, R.D., CALDWELL, J.P., VITT, L.J. \& GIBBONS, J.W. 1991. Declining amphibian populations: the problem of separating human impacts from the natural fluctuations. Science 253:892-895. http://dx.doi.org/10.1126/science.253.5022.892

PONSSA, M.L. 2008. Cladistic analysis and osteological descriptions of the frog species in the Leptodactylus fuscus species group (Anura, Leptodactylidae). J. Zool. Syst. Evol. Res. 46:249-266. http://dx.doi. org/10.1111/j.1439-0469.2008.00460.x 
POUNDS, J.A., BUSTAMANTE, M.R., COLOMA, L.A., CONSUEGRA, J.A., FOGDEN, M.P.L., FOSTER, P.N., LA MARCA, E., MASTERS, K.L., MERINO-VITERI, A., PUSCHENDORF, R., RON, S.R., SÁNCHEZ-AZOFEIFA, A., STILL, C.J. \& YOUNG, B.E. 2006. Widespread amphibian extinctions from epidemic disease driven by global warming. Nature 439:161-167. http://dx.doi.org/10.1038/nature04246

POUNDS, J.A., BUSTAMANTE, M.R., COLOMA, L.A., CONSUEGRA, J.A., FOGDEN, M.P.L., FOSTER, P.N., LA MARCA, E., MASTERS, K.L., MERINO-VITERI, A., PUSCHENDORF, R., RON, S.R., SÁNCHEZ-AZOFEIFA, A., STILL, C.J. \& YOUNG, B.E. 2007. Global warming and amphibian losses; the proximate cause of frog declines? (Reply). Nature 447:E3-E4.

PRADO, V.H.M., SILVA, F.R., BORGES, R.E., TOGNOLO, T.T. \& ROSSAFERES, D.C. 2008. Amphibia, Anura, Hylidae, Phyllomedusa azurea: Distribution extension. Check List 4:55-56.

PRAMUK, J.B. 2006. Phylogeny of south American Bufo (Anura: Bufonidae) inferred from combined evidence. Zool. J. Linn. Soc. 146: 407-452. http:// dx.doi.org/10.1111/j.1096-3642.2006.00212.x

PRAMUK, J.B., ROBERTSON, T., SITES JUNIOR, J.W. \& NOONAN, B.P. 2007. Around the world in 10 million years: biogeography of the nearly cosmopolitan true toads (Anura: Bufonidae). Glob. Ecol. Biogeogr. 17:72-83.

PURVIS, A., GITTLEMAN, J.L. \& BROOKS, T. (eds.). 2005. Phylogeny and conservation. Cambridge University Press, 428p.

RELYEA, R.A., SCHOEPPNER, N.M. \& HOVERMAN, J.T. 2005. Pesticides and amphibians: the importance of community context. Ecol. Appl. 15:1125-1134. http://dx.doi.org/10.1890/04-0559

RESETARITIS JUNIOR, W.J. \& BERNARDO, J. (eds.). 1998. Experimental ecology: issues and perspectives. Oxford University Press, 343p.

RODRIGUES, R.R., JOLY, C.A., BRITO, M.C.W, PAESE, A., METZGER, J.P., CASATTI, L., NALON, M.A., MENEZES, N., IVANAUSKAS, N.M., BOLZANI, V. \& BONONI, V.L.R. 2008. Diretrizes para a conservação da biodiversidade no estado de São Paulo. Instituto de Botânica; FAPESP, São Paulo.

ROMERO, G.Q., NOMURA, F., GONCALVES, A.Z., DIAS, N.Y.N., MERCIER, H., CONFORTO, E.C. \& ROSSA-FERES, D.C. 2010. Nitrogen fluxes from treefrogs to tank epiphytic bromeliads: an isotopic and physiological approach. Oecologia 162:941-949. http://dx.doi. org/10.1007/s00442-009-1533-4

ROSSA-FERES, D.C. 1997. Ecologia de uma comunidade de anfíbios anuros da região noroeste do Estado de São Paulo: microhabitat, sazonalidade, dieta e nicho multidimensional. Tese de doutorado, UNESP, Rio Claro.

ROSSA-FERES, D.C., MARTINS, M., MARQUES, O.A.V., MARTINS, I.A., SAWAYA, R.J. \& HADDAD, C.F.B. 2008. Herpetofauna. In Diretrizes para a conservação e restauração da biodiversidade no Estado de São Paulo (R.R. Rodrigues, C.A. Joly, M.C.W. de Brito, A. Paese, J.P. Metzger, L. Casatti, M.A. Nalon, N. Menezes, N.M. Ivanauskas, V. Bolzani, V.L.R. Bononi, coords.). Instituto de Botânica; FAPESP, São Paulo, p.83-94.

SANTOS, T.G., ROSSA-FERES, D.C. \& CASATTI, L. 2007. Diversidade e distribuição espaço-temporal de anuros em região com pronunciada estação seca no sudeste do Brasil. Iheringia 97:37-49. http://dx.doi. org/10.1590/S0073-47212007000100007

SANTOS, T.G., VASCONCELOS, T.S., ROSSA-FERES, D.C., HADDAD, C.F.B. 2009. Anurans of a seasonally dry tropical forest: Morro do Diabo State Park, São Paulo state, Brazil. J. Nat. Hist. 43:973-993.

SÃO PAULO (Estado). Decreto n ${ }^{\circ} 53.494$, de 2 de outubro de 2008. Declara as espécies da fauna silvestre ameaçadas, as quase ameaçadas, as colapsadas, sobrexplotadas, ameaçadas de sobrexplotação e com dados insuficientes para avaliação no Estado de São Paulo e dá providências correlatas. Diário Oficial do Estado, v.118, n.187, 3 de outubro de 2008.

SAWAYA, R.J. \& HADDAD, C.F.B. 2006. Amphibia, Anura, Stereocyclops parkeri: distribution extension, new state record, geographic distribution map. Check List 2:74-76.
SCHIESARI, L., GRILLITSCH, B. \& GRILLITSCH, H. 2007. Biogeographic biases in research and their consequences for linking amphibian declines to pollution. Cons. Biol. 21:465-471. http://dx.doi.org/10.1111/j.15231739.2006.00616.x

SCHIESARI, L., WERNER, E., KLING, G.W. 2009. Carnivory and resourcebased niche differentiation in anuran larvae: implications for food web and experimental ecology. Fresh. Biol. 54:572-586. http://dx.doi. org/10.1111/j.1365-2427.2008.02134.x

SCHWARTZ, C.A., CASTRO, M.S., PIRES, J.R., OSMINDO R., MACIEL, N.M., SCHWARTZ, E.F. \& SEBBEN, A. 2007. Princípios bioativos de pele de anfíbios: panorama atual e perspectivas In Herpetologia no Brasil II (L.B. Nascimento \& M.E. Oliveira, orgs.). Sociedade Brasileira de Herpetologia, Belo Horizonte, p.146-168.

SILVA, F.R., PRADO, V.H.M., VASCONCELOS, T.S., SANTOS, T.G. \& ROSSA-FERES, D.C. 2009. Amphibia, Anura, Microhylidae, Chiasmocleis albopunctata: filling gap and geographic distribution map. Check List 5:314-316.

SILVA, F.R., PRADO, V.H.M. \& ROSSA-FERES, D.C. 2010. Amphibia, Anura, Hylidae, Dendropsophus melanargyreus (Cope, 1887): Distribution extension, new state record and geographic distribution map. CheckList 6:402-404.

SILVANO, D.L. \& SEGALLA, M.V. 2005. Conservação de anfíbios no Brasil. Megadiversidade 1(1):79-86.

SKELLY, D.K., YUREWICZ, K.L., WERNER, E.E. \& RELYEA, R.A. 2003. Quantifying decline and distributional change in amphibians. Cons. Biol. 17:744-751. http://dx.doi.org/10.1046/j.1523-1739.2003.02009.x

SOCIEDADE BRASILEIRA DE HERPETOLOGIA - SBH. 2010. Lista de espécies de anfíbios e répteis do Brasil. Sociedade Brasileira de Herpetologia, São Paulo. http://www.sbherpetologia.org.br/ (último acesso em 04/07/2010.)

SPECIESLINK. Sistema distribuído de Informação que integra, em tempo real, dados primários de coleções científicas. http://splink.cria.org.br/ (último acesso em 15/06/2010).

THOMÉ, M.T., ZAMUDIO, K.R., GIOVANELLI, J.G.R., HADDAD, C.F.B., BALDISSERA JUNIOR., F.A. \& ALEXANDRINO, J. 2010. Phylogeography of endemic toads and post-Pliocene persistence of the Brazilian Atlantic Forestry. Mol. Phylogenet. Evol. 55:1018-1031. http:// dx.doi.org/10.1016/j.ympev.2010.02.003

TOLEDO, L.F., BRITTO, F.B., ARAUJO, O.G.S., GIASSON, L.O.M. \& HADDAD, C.F.B. 2006a. The occurrence of Batrachochytrium dendrobatidis in Brazil and the inclusion of 17 new cases of infection. South Am. J. Herpetol. 1:185-191. http://dx.doi.org/10.2994/18089798(2006)1[185:TOOBDI]2.0.CO;2

TOLEDO, L.F., HADDAD, C.F.B., CARNAVAL, A.C.O.Q. \& BRITTO, F.B. 2006b. A Brazilian anuran (Hylodes magalhaesi: Leptodactylidae) infected by Batrachochytrium dendrobatidis: a conservation concern. Amphibian \& Reptile Conservation 4(1):17-21.

TOLEDO, L.F., GARCIA, P.C.A., LINGNAU, R. \& HADDAD, C.F.B. 2007. A new species of Sphaenorhynchus (Anura: Hylidae) from Brazil. Zootaxa 1658:57-68.

TOLEDO, L.F., BRASILEIRO, C.A., ARAÚJO, O.G.S. \& HADDAD, C.F.B. 2008. Amphibia, Anura, Hylidae, Bokermannohyla izecksohni: distribution extension. Check List 4:442-444.

TOLEDO, L.F. 2010. A new species of Elachistocleis (Anura; Microhylidae) from the Brazilian Amazon. Zootaxa 2496:63-68.

TOLEDO, L.F., SIQUEIRA, S., DUARTE, T.C., VEIGA-MENONCELLO, A.C.P., RECCO-PIMENTEL, S.M. \& HADDAD, C.F.B. 2010. Description of a new species of Pseudopaludicola Miranda-Ribeiro, 1926 from the state of São Paulo, Southeastern Brazil (Anura, Leiuperidae). Zootaxa 2496:38-48.

TRUEB, L. \& MASSEMIN, D. 2001. The osteology and relationships of Pipa aspera (Amphibia: Anura: Pipidae), with notes on its natural history in French Guiana. Amphib-Reptilia 22:33-54. 
VAN BOCXLAER, I., LOADER, S.P., ROELANTS, K., BIJU, S.D., MENEGON, M. \& BOSSUYT, F. 2010. Gradual adaptation toward a range-expansion phenotype initiated the global radiation of toads. Science 327:679-682. http://dx.doi.org/10.1126/science.1181707

VAN DER MEIJDEN, A., VENCES, M., HOEGG, S., BOISTEL, R., CHANNING, A. \& MEYER, A. 2007. Nuclear gene phylogeny of narrowmouthed toads (Family: Microhylidae) and a discussion of competing hypotheses concerning their biogeographical origins. Mol. Phylogenet. Evol. 44:1017-1030. http://dx.doi.org/10.1016/j.ympev.2007.02.008

VASCONCELOS, T.S. \& ROSSA-FERES, D.C. 2005. Diversidade, distribuição espacial e temporal de anfíbios anuros (Amphibia, Anura) na região noroeste do Estado de São Paulo. Biota Neotrop. 5(2):1-14. http:// www.biotaneotropica.org.br/v5n2/pt/abstract?article+BN01705022005 (último acesso em 18/06/2010).

VASCONCELOS, T.S., SANTOS, T.G., ROSSA-FERES, D.C. \& HADDAD, C.F.B. 2009. Influence of the environmental heterogeneity of breeding ponds on anuran assemblages from Southeastern Brazil. Can. J. Zool. 87:699-707. http://dx.doi.org/10.1139/Z09-058

VASCONCELOS, T.S., SANTOS, T.G., HADDAD, C.F.B. \& ROSSAFERES, D.C. 2010. Climatic variables and altitude as predictors of anuran species richness and number of reproductive modes in Brazil. J. Trop. Ecol. 26:423-432. http://dx.doi.org/10.1017/S0266467410000167

VÁZQUEZ, D.P. \& GITTLEMAN, J.L. 1998. Biodiversity conservation: does phylogeny matter? Curr. Biol. 8:R379-R381. http://dx.doi.org/10.1016/ S0960-9822(98)70242-8

VERDADE, V.K. \& RODRIGUES, M.T. 2008. On the identity of Cycloramphus jordanensis Heyer, 1983 (Anura: Cycloramphidae). Herpetologica 64:452-457. http://dx.doi.org/10.1655/08-011R2.1

VERDADE, V.K, CARNAVAL, A.C.O.Q., RODRIGUES, M.T.U., SCHIESARI, L., PAVAN, D. \& BERTOLUCI, J. in press. Decline of amphibians in Brazil. In Biology of amphibians. Regional assessment of decline in amphibians (H. Heatwole \& M. Wilkinson, orgs.). Surrey Beatty and Sons, Chipping Norton, Australia.

WAKE, D.B. 1991. Declining amphibian populations. Science 250:860. http:// dx.doi.org/10.1126/science.253.5022.860

WELLBORN, G.A., SKELLY, D.K. \& WERNER, E.E. 1996. Mechanisms creating community structure across a freshwater habitat gradient. Ann. Rev. Ecol. Evol. Syst. 27:337-363. http://dx.doi.org/10.1146/annurev. ecolsys.27.1.337
WERNER, E.E. 1992. Individual behavior and higher-order species interactions. Am. Nat. 140:5-32. Suppl. http://dx.doi.org/10.1086/285395

WERNER, E.E. 1998. Ecological experiments and a research program in community ecology. In Experimental ecology: issues and perspective (W.J. Resetarits \& J. Bernardo, eds.). Oxford University Press, Oxford, p3-26.

WERNER, E.E. \& PEACOR, S.D. 2003. A review of trait-mediated indirect interactions in ecological communities. Ecology 84(5):1083-1100. http:// dx.doi.org/10.1890/0012-9658(2003)084[1083:AROTII]2.0.CO;2

WERNER, E.E., SKELLY, D.K., RELYEA, R.A. \& YUREWICZ, K.L. 2007. Amphibian species richness across environmental gradients. Oikos 116:1697-1712. http://dx.doi.org/10.1111/j.0030-1299.2007.15935.x

WIENS, J.J., GRAHAM, C.H., MOEN, D.S., SMITH, S.A. \& REEDER, T.W. 2006. Evolutionary and ecological causes of the latitudinal diversity gradient in hylid frogs: treefrog tree unearth the roots of high tropical diversity. Am. Nat. 168:579-596. http://dx.doi.org/10.1086/507882

WIENS, J.J., KUCZYNSKI, C.A., DUELLMAN, W.E. \& REEDER, T.W. 2007. Loss and re-evolution of complex life cycles in marsupial frogs: does ancestral trait reconstruction mislead? Evolution 61:1886-1899. http://dx.doi.org/10.1111/j.1558-5646.2007.00159.x

WIENS, J.J., KUCZYNSKI, C.A., HUA, X. \& MOEN, D.S. 2010. An expanded phylogeny of treefrogs (Hylidae) based on nuclear and mitochondrial sequence data. Mol. Phylogenet. Evol. 55:871-882. http:// dx.doi.org/10.1016/j.ympev.2010.03.013

WILBUR, H.M. 1976. Density-dependent aspects of metamorphosis in Ambystoma and Rana sylvatica. Ecology 57:1289-1296. http://dx.doi. org/10.2307/1935053

WILBUR, H.M. 1987. Regulation of structure in complex systems: experimental temporary pond communities. Ecology 68:1437-1452.

WILBUR, H.M. 1997. Experimental ecology of food webs: complex systems in temporary ponds. Ecology 78(8):2279-2302. http://dx.doi. org/10.1890/0012-9658(1997)078[2279:EEOFWC]2.0.CO;2

ZINA, J., ENNSER, J., PINHEIRO, S.C.P., HADDAD, C.F.B. \& TOLEDO, L.F. 2007. Taxocenose de anuros de uma mata semidecídua do interior do Estado de São Paulo e comparações com outras taxocenoses do Estado, sudeste do Brasil. Biota Neotrop. 7(2):49-58. http://www. biotaneotropica.org.br/v7n2/pt/abstract?article+bn00607022007 (último acesso em 20/06/2010). 


\section{Apêndices}

Apêndice 1. Lista de projetos no âmbito ou não do Programa Biota/FAPESP, que contribuíram para o diagnóstico da riqueza e distribuição de espécies de anfíbios no Estado de São Paulo. Os projetos estão ordenados pelo nome do coordenador.

Appendix 1. List of projects of the Biota/FAPESP Program or not, which contributed to the diagnosis of the richness and distribution of amphibian species in São Paulo. The projects are listed by the name of the coordinator.

\begin{tabular}{|c|c|c|c|}
\hline & Projeto & Coordenador & Instituição sede \\
\hline 1 & Diversidade de anfíbios anuros do Estado de São Paulo & Célio F.B. Haddad & UNESP/Rio Claro \\
\hline 2 & Especiação de anfíbios anuros em ambientes de altitude & Célio F.B. Haddad & UNESP/Rio Claro \\
\hline 3 & $\begin{array}{l}\text { Diversidade e biogeografia de anfíbios anuros em ilhas do } \\
\text { Estado de São Paulo }\end{array}$ & Cinthia A. Brasileiro & UNIFESP/Diadema \\
\hline 4 & $\begin{array}{l}\text { Biodiversidade, biologia e comportamento reprodutivo de } \\
\text { anfíbios anuros na região Serra da Mantiqueira, vale do Paraíba } \\
\text { paulista, Estado de São Paulo }\end{array}$ & Itamar A. Martins & UNITAU/Taubaté \\
\hline 5 & $\begin{array}{l}\text { Comunidades de anfíbios anuros em diferentes gradientes } \\
\text { altitudinais na região da Serra da Mantiqueira, SP }\end{array}$ & Itamar A. Martins & UNITAU/Taubaté \\
\hline 6 & $\begin{array}{l}\text { Anurofauna de quatro florestas do Estado de São Paulo: } \\
\text { composição de espécies, distribuição espacial, padrões anuais } \\
\text { de reprodução e monitoramento de populações }\end{array}$ & Jaime A. Bertoluci & USP/Piracicaba \\
\hline 7 & $\begin{array}{l}\text { Biogeografia, filogeografia e padrões de diversificação de anuros } \\
\text { comuns na Mata Atlântica do Brasil }\end{array}$ & João Alexandrino & UNESP/Rio Claro \\
\hline 8 & $\begin{array}{l}\text { Impactos da expansão do agronegócio da cana-de-açúcar sobre } \\
\text { comunidades aquáticas* }\end{array}$ & Luis C. Schiesari & USP/São Paulo \\
\hline 9 & $\begin{array}{l}\text { Conservação de anfíbios e répteis neotropicais: aspectos } \\
\text { ecológicos e evolutivos }\end{array}$ & Márcio R. C. Martins & USP/São Paulo \\
\hline 10 & $\begin{array}{l}\text { Fauna e flora de fragmentos florestais remanescentes no noroeste } \\
\text { paulista: base para estudos de conservação da biodiversidade }\end{array}$ & Orlando Necchi Júnior & $\begin{array}{l}\text { UNESP/São José do } \\
\text { Rio Preto }\end{array}$ \\
\hline 11 & $\begin{array}{l}\text { Diversidade, distribuição e conservação da herpetofauna do } \\
\text { Estado de São Paulo }\end{array}$ & Ricardo J. Sawaya & $\begin{array}{l}\text { Instituto Butantan/São } \\
\text { Paulo }\end{array}$ \\
\hline 12 & Phylogenetic studies on hylid frogs: From the trunk to the tips & Julian Faivovich & UNESP/Rio Claro \\
\hline
\end{tabular}

*Financiado pelo Edital FAPESP BIOEN.

Apêndice 2. Referências complementares sobre anfíbios do Estado de São Paulo.

Appendix 2. Additional references about the amphibians of the State of São Paulo.

AGUIAR-DE-DOMENICO, E. 2008. Herpetofauna do Mosaico de Unidades de Conservação do Jacupiranga (SP). Dissertação de Mestrado, Universidade de São Paulo, São Paulo.

ALMEIDA, S.C., MAFFEI, F., ROLIM, D.C., UBAID, F.K. \& JIM, J. 2008. Amphibia, Anura, Hylidae, Sphaenorhynchus caramaschii: Distribution extension in state of São Paulo, Brazil. Check List 4(4):439-441.

ANTUNES, A.P. 2007. Descrição taxonômica e história natural de uma nova espécie de Hypsiboas da Mata Atlântica do alto da serra de Paranapiacaba, estado de São Paulo (Amphibia, Anura, Hylidae). Dissertação de Mestrado, Universidade Estadual Paulista Júlio de Mesquita Filho, Rio Claro.

ARAUJO, C.O. 1997. Levantamento da fauna de anuros do Parque Anhanguera São Paulo, SP. Relatório de iniciação à Pesquisa II, Universidade de São Paulo, São Paulo.

BERNARDE, P.S. \& KOKUBUM, M.N.C. 1999. Anurofauna do município de Guararapes, Estado de São Paulo, Brasil. Acta Biol. Leopol., 21(1):89-97.

BERTOLUCI, J.A. 2001. Anfíbios anuros. In Intervales: fundação para a conservação e produção florestal do estado de São Paulo (C. Leonel, ed.). Editora Secretaria do Meio Ambiente; Fundação Florestal, São Paulo, p.159-168.

BERTOLUCI, J. \& RODRIGUES, M.T. 2002. Utilização de habitats reprodutivos e micro-habitats de vocalização em uma taxocenose de anuros (Amphibia) da Mata Atlântica do sudeste do Brasil. Pap. Avul. Zool. 42(11):287-297. http://dx.doi.org/10.1590/S0031-10492002001100001

BRASILEIRO, C.A. 2004. Diversidade de anfíbios anuros em área de cerrado no Estado de São Paulo. Tese de Doutorado, Universidade de São Paulo, São Paulo.

BRASILEIRO, C.A., MARTINS, I.A. \& JIM, J. 2008. Amphibia, Anura, Cycloramphidae, Odontophrynus moratoi: Distribution extension and advertisement call. Check List 4(4):382-385.

BRASILEIRO, C.A.; SAWAYA, R.J.; KIEFER, M.C. \& MARTINS, M. 2005. Anfíbios de um fragmento de Cerrado aberto do sudeste do Brasil. Biota Neotrop. 5(2): http://www.biotaneotropica.org.br/v5n2/pt/abstract?article+BN00405022005 (último acesso em 15/06/2010).

BRUSCAGIN, R.T. 2010. Diversidade de anfíbios anuros e lagartos de serapilheira em uma paisagem fragmentada de Ribeirão Grande, São Paulo. Dissertação de Mestrado, Escola Superior de Agricultura Luiz de Queiroz, Piracicaba.

CARAMASCHI, U. 1981. Variação estacional, distribuição espacial e alimentação de populacões de hilídeos na represa do rio Pardo (Botucatu, SP) (Amphibia, Anura, Hylidae). Dissertação de Mestrado, Universidade Estadual de Campinas, Campinas.

CARDOSO, A.J. 1981. Organização espacial e temporal na reprodução e vida larvária em uma comunidade no sudeste do Brasil (Amphibia, Anura). Dissertação de Mestrado, Universidade Estadual de Campinas, Campinas. 
Rossa-Feres, D.C. et al.

CARMONA, R.U. 2007. Estudo de comunidade de anfíbios e répteis em um fragmento de Mata Atlântica e em áreas perturbadas no estado de São Paulo: subsídios para conservação e manejo de áreas protegidas. Dissertação de Mestrado, Escola Superior de Agricultura Luiz de Queiroz, Piracicaba.

CARVALHO-JUNIOR, R.R., KLEINSORGE, J.M.D. \& FUSINATTO, L.A. 2010. Amphibia, Anura, Cycloramphidae, Odontophrynus moratoi Jim and Caramaschi, 1980: Filling gaps. Discovery of a new population in the state of SãoPaulo, southeastern Brazil. Check List 6(1):36-37.

CENTENO, F. 2008. Diversidade e uso do ambiente pelos anfíbios e répteis da Ilha de São Sebastião, Ilhabela, SP. Dissertação de Mestrado, Universidade de São Paulo, São Paulo.

CICCHI, P.J.P., SERAFIM, H., SENA, M.A., CENTENO, F.C. \& JIM, J. 2009. Atlantic Rainforest herpetofauna of Ilha Anchieta, an island on municipality of Ubatuba, southeastern Brazil. Biota Neotrop. 9(2): http://www.biotaneotropica.org.br/v9n2/en/abstract?inventory+bn01009022009 (último acesso em $15 / 06 / 2010)$.

CONDEZ, T.C. 2008. Efeitos da fragmentação da floresta na diversidade e abundância de anfíbios anuros e lagartos de serapilheira em uma paisagem do Planalto Atlântico de São Paulo. Dissertação de Mestrado, Universidade de São Paulo, São Paulo.

CONDEZ, T.H., SAWAYA, R.J. \& DIXO, M. 2009. Herpetofauna of the Atlantic Forest remnants of Tapiraí and Piedade region, São Paulo state, southeastern Brazil. Biota Neotrop. 9(1): http://www.biotaneotropica.org.br/v9n1/en/abstract?inventory+bn01809012009 (último acesso em 15/06/2010).

CONTE, C.E. 2010. Diversidade de anfíbios da floresta com Araucária. Tese de Doutorado, Universidade Estadual Paulista Julio de Mesquita Filho, São José do Rio Preto.

CONTE, C.E., NOMURA, F., MACHADO, R.A., KWET, A., LINGNAU, R., ROSSA-FERES, D.C. 2010. Novos registros na distribuição geográfica de anuros na Floresta com Araucária e considerações sobre suas vocalizações. Biota Neotrop. 2:201-224. http://dx.doi.org/10.1590/S1676-06032010000200024

DIXO, M. 2005. Diversidade de sapos e lagartos de serapilheira numa paisagem fragmentada do Planalto Atlântico de São Paulo. Tese de Doutorado, Universidade de São Paulo, São Paulo.

DIXO, M. \& VERDADE, V.K. 2006. Leaf litter herpetofauna of the Reserva Florestal de Morro Grande, Cotia (SP). Biota Neotrop. 6(2): http://www. biotaneotropica.org.br/v6n2/pt/abstract?article+bn00806022006 (último acesso em 12/05/2010).

DUELLMAN, W.E. \& TRUEB, L. 1994. Biology of Amphibians. McGraw-Hill, Baltimore; London.

ETEROVICK, P.C. 1998. Estruturação espacial e temporal de uma comunidade de anuros (Amphibia) na Serra do Cipó, Minas Gerais. Dissertação de Mestrado, Universidade Estadual de Campinas, Campinas.

FORLANI, M.C., BERNARDO, P.H., HADDAD, C.B.F. \& ZAHER, H. 2010. Herpetofauna do Parque Estadual Carlos Botelho, São Paulo, Brasil. Biota Neotrop. 10(3): http://www.biotaneotropica.org.br/v10n3/pt/abstract?article+bn00210032010 (último acesso em 15/06/2010). http://dx.doi.org/10.1590/ S1676-06032010000400031

GIARETTA, A.A. 1994. Utilização de recursos e potencial reprodutivo dos leptodactilídeos (Amphibia-Anura) de uma floresta semidecídua de altitude no sudeste do Brasil. Dissertação de Mestrado, Universidade Estadual de Campinas, Campinas.

GIARETTA, A.A. 1999. Diversidade e densidade de anuros de serapilheira num gradiente altitudinal na mata costeira. Tese de Doutorado, Universidade Estadual de Campinas, Campinas.

GIARETTA, A.A., SAWAYA, R.J., MACHADO, G., ARAÚJO, M.S., FACURE, K.G., MEDEIROS, K.G. \& NUNES, R. 1997. Diversity and abundance of litter frogs at altitudinal sites at Serra do Japi, southeastern Brazil. Rev. Bras. Zool. 14(2):341:346. http://dx.doi.org/10.1590/S0101-81751997000200008

GIARETTA, A.A., FACURE, K.G., SAWAYA, R.J., MEYER, J.H.M. \& CHEMIN, N. 1999. Diversity and abundance of litter frogs in a montane Forest in southeastern Brazil: seasonal and altitudinal. Biotropica 31(4):669-674. http://dx.doi.org/10.1111/j.1744-7429.1999.tb00416.x

GIASSON, L.O.M. 2008. Atividade sazonal e uso do ambiente por anfíbios da Mata Atlântica no alto da Serra do Mar. Tese de Doutorado, Universidade Estadual Paulista Júlio de Mesquita Filho, Rio Claro.

GIOVANELLI, J.G.R. 2009. Modelagem de nicho ecológico de anuros da Mata Atlântica. Dissertação de Mestrado, Universidade Estadual Paulista Júlio de Mesquita Filho, Rio Claro.

GOMES, F.B.R. 2009. Uso de habitats e ecomorfologia de girinos na Serra da Mantiqueira, SP. Dissertação de Mestrado, Universidade Estadual Paulista Júlio de Mesquita Filho, Rio Claro.

GRESSLER, E., AGUIRRE, A.G. \& HADDAD, C.F.B. 2008. Amphibia, Anura, Amphignathodontidae, Gastrotheca albolineata: distribution extension, new state, and new altitudinal records. Check List 4(1):31-32.

GUIX, J.C., LORENTE, G., MONTORI, A., CARRETERO, M.A. \& SANTOS, X. 2000. Una nueva área de elevada riqueza de anuros en el bosque lluvioso atlántico de Brasil. Bol. Asoc. Herpetol. Esp. 11(2):100-105.

HADDAD, C.F.B. \& SAZIMA, I. 1992. Anfíbios anuros da Serra do Japi. In História Natural da Serra do Japi: ecologia e preservação de uma área florestal no sudeste do Brasil (L.P.C. Morellatto, ed.). Editora UNICAMP; FAPESP, Campinas, p. 188-211.

HADDAD, C.F.B., TOLEDO, L.F. \& PRADO, C.P.A. 2008. Anfíbios da Mata Atlântica: guia dos anfíbios anuros da Mata Atlântica. Editora Neotropica, São Paulo.

HARTMANN, M.T. 2004. Biologia reprodutiva de uma comunidade de anuros (Amphibia) na Mata Atlântica (Picinguaba, Ubatuba, SP). Tese de Doutorado, Universidade Estadual Paulista Júlio de Mesquita Filho, Rio Claro.

HEYER, W.R., RAND, A.S., CRUZ, C.A.G., PEIXOTO, O.L. \& NELSON, C.E. 1990. Frogs of Boracéia. Arq. Zool. 31(4):231-410.

MAFFEI, F., UBAID, F.K., ALMEIDA, S.C., ROLIM, D.C., SCARPELLINI-JUNIOR., D.G., MOYA, G.M., SPIRANDELLI-CRUZ, E.F. \& JIM, J. 2009. Amphibia, Anura, Hylidae, Dendropsophus microps (Peters, 1872): distribution extension in state of São Paulo, Brazil and first record in Cerrado domain. Check List 5(4):776-779.

MAFFEI, F., UBAID, F.K. \& JIM, J. 2010. Predation of herps by spiders (Araneae) in the Brazilian Cerrado. Herpetol. Notes 3:167-170.

MALAGOLI, L.R. 2007. Anfíbios do Município de São Paulo. In Fauna silvestre: quem são e onde vivem os animais na metrópole paulistana (A.F.A. Magalhães \& M.K. Vasconcellos, orgs.). Imprensa Oficial do Estado de São Paulo, São Paulo, p.64-103.

MALAGOLI, L.R. 2008. Anfíbios do município de São Paulo: histórico, conhecimento atual e desafios para a conservação. In Além do concreto: contribuições para a proteção da biodiversidade paulistana (L.R. Malagoli, F.B. Bajesteiro \& M. Whately, orgs.). Instituto Sócio Ambiental, São Paulo, p.204-231.

MARQUES, R.M., COLAS-ROSAS, P.F., TOLEDO, L.F. \& HADDAD, C.F.B. 2006. Amphibia, Anura, Bufonidae, Melanophryniscus moreirae: distribution extension. Check List 2(1):68-69. 
Anfíbios do Estado de São Paulo

MELO, G.V., ROSSA-FERES, D.C. \& JIM, J. 2007. Temporal variation in calling site use in a community of anurans in Botucatu, São Paulo State, Brazil Biota Neotrop. 7(2): http://www.biotaneotropica.org.br/v7n2/pt/abstract?article+bn01707022007 (último acesso em 12/06/2010).

MORAES, R.A., SAWAYA, R.J. \& BARRELA, W. 2007. Composition and diversity of Anuran Amphibians in two Atlantic Forest environments in Southeastern Brazil, Parque Estadual Carlos Botelho, São Paulo, Brazil. Biota Neotrop. 7(2): http://www.biotaneotropica.org.br/v7n2/pt/abstract?article+bn00307022007 (último acesso em 12/06/2010).

NOMURA, F. 2008. Padrões de diversidade e estrutura de taxocenoses de anfíbios anuros: análise em multi-escala espacial. Tese de Doutorado, Universidade Estadual Paulista Júlio de Mesquita Filho, Rio Claro.

OLIVEIRA, S.H. 2004. Diversidade de anuros de serapilheira em fragmentos de Floresta Atlântica e plantio de Eucalyptus saligna no município de Pilar do Sul, SP. Dissertação de Mestrado, Escola Superior de Agricultura Luiz de Queiroz, Piracicaba.

OLIVEIRA, T.M. 2008. Uso de hábitat, micro-hábitat e coexistênica com predadores em taxocenoses de girinos de anuros no noroeste paulista. Tese de Doutorado, Universidade Estadual Paulista Júlio de Mesquita Filho, São José do Rio Preto.

PEACOR, S.D. \& WERNER, E.E. 2001. The contribution of trait-mediated indirect effects to the net effects of a predator. Proceedings of the National Academy of Sciences 98:3904-3908. PMid:11259674. PMCid:31151. http://dx.doi.org/10.1073/pnas.071061998

PIMENTA, B.V.S., BÉRNILS, R.S. \& POMBAL JUNIOR., J.P. 2007. Amphibia, Anura, Brachycephalidae, Brachycephalus hermogenesi: Filling gap and geographic distribution map. Check List 3(3):277-279.

PINHEIRO, S.C.P. 2010. Anurofauna de serapilheira de diferentes formações vegetais ao longo de um gradiente altitudinal no Parque Estadual da Ilha do Cardoso, município de Cananéia, SP. Dissertação de Mestrado, Escola Superior de Agricultura Luiz de Queiroz, Piracicaba.

POMBAL, J.P. 1997. Distribuição espacial e temporal de anuros (Amphibia) em uma poça permanente na serra de Paranapiacaba, sudeste do Brasil. Rev. Bras. Zool. 57(4):583-594.

POMBAL-JUNIOR., J.P. \& GORDO, M. 2004. Anfíbios da Juréia. In Estação Ecológica Juréia-Itatins. Ambiente fisíco, flora e fauna. (O.A.V. Marques \& W. Duleba, orgs.) Holos Editora, Ribeirão Preto, p.243-256.

PRADO, V.H.M. 2009. Uso de remanescentes florestais pela anurofauna da região noroeste do estado de São Paulo. Tese de Doutorado, Universidade Estadual Paulista Julio de Mesquita Filho, São José do Rio Preto.

RAMOS, J.Z.P. 2010. Estudo comparativo das taxocenoses de anuros de quatro Municípios do Lagamar Paulista. Tese de Doutorado, Universidade Estadual Paulista Júlio de Mesquita Filho, Rio Claro.

RELYEA, R.A., SCHOEPPNER, N.M. \& HOVERMAN, J.T. 2005. Pesticides and amphibians: the importance of community context. Ecol. Appl. 15:11251134. http://dx.doi.org/10.1890/04-0559

RIBEIRO-JÚNIOR, J.W. \& BERTOLUCI, J. 2009. Anurans of the cerrado of the Estação Ecológica and the Floresta Estadual de Assis, southeastern Brazil. Biota Neotrop. 9(1): http://www.biotaneotropica.org.br/v9n1/en/abstract?inventory+bn02709012009 (último acesso em 12/06/2010).

RIBEIRO, R.S., EGITO, G.T.B.T. \& HADDAD, C.F.B. 2005. Chave de identificação: anfíbios anuros da vertente de Jundiaí da Serra do Japi, Estado de São Paulo. Biota Neotrop. 5(2): http://www.biotaneotropica.org.br/v5n2/pt/abstract?identification-key+bn03005022005 (último acesso em 12/06/2010).

RIBEIRO, R.S. 2006. Ecologia alimentar das quatro espécies dominantes da anurofauna de serapilheira em um gradiente altitudinal na Ilha de São Sebastião, SP. Universidade Estadual Paulista Júlio de Mesquita Filho. Dissertação de Mestrado, Rio Claro.

ROLIM, D.C., MAFFEI, F., SENE, R.K., MEDOLAGO, C.A.B., VERNINI, T.H., UBAID, F.K. \& JIM, J. 2008. Amphibia, Anura, Hylidae, Dendropsophus anceps: distribution extension in state of São Paulo, Brazil. Check List 4(3):358-361.

ROSSA-FERES, D.C. \& NOMURA, F. 2006. Caracterização e chave taxonômica para girinos (Amphibia: Anura) da região noroeste do estado de São Paulo, Brasil. Biota Neotrop. 5(2): http://www.biotaneotropica.org.br/v6n1/pt/abstract?identification-key+bn00706012006 (último acesso em 12/06/2010). http:// dx.doi.org/10.1590/S0101-81752001000200015

ROSSA-FERES, D.C. 1989. Distribuição sazonal e espacial de girinos em corpos d'água na região de Botucatu, São Paulo (Amphibia. Anura). Dissertação de Mestrado, Universidade Estadual de Campinas, Campinas.

ROSSA-FERES, D.C. \& JIM, J. 2001. Similaridade do sítio de vocalização em uma comunidade de anfíbios anuros na região noroeste do Estado de São Paulo, Brasil. Rev. Bras. Zool. 18 (2): 439-454.

SANTOS, T.G. 2009. Diversidade de Anuros (Amphibia) do Parque Estadual Morro do Diabo, SP. Tese de Doutorado, Universidade Estadual Paulista Júlio de Mesquita Filho, Rio Claro.

SAWAYA, R.J. 1999. Diversidade, densidade e distribuição altitudinal da anurofauna de serapilheira da Ilha de São Sebastião, SP. Dissertação de Mestrado, Universidade de São Paulo, São Paulo.

SERAFIM, H., CICCHI, P.J.P., IENNE, S. \& JIM, J. 2008. Anurofauna de remanescentes de floresta Atlântica do município de São José do Barreiro, estado de São Paulo, Brasil. Biota Neotrop. 8(2): http://www.biotaneotropica.org.br/v8n2/pt/abstract?article+bn01008022008 (último acesso em 12/06/2010).

SIERRA-RAMÍREZ, N.M. 1998. Análise comparativa entre comunidades de anfíbios anuros do sudeste brasileiro e uma região dos andes baixos da Venezuela. Tese de doutorado, Universidade Estadual de Campinas, Campinas.

SILVA, F.R. 2007. A importância de fragmentos florestais na diversidade de anfíbios anuros em Icém, região noroeste do Estado de São Paulo. Dissertação de mestrado, Universidade Estadual Paulista Júlio de Mesquita Filho, São José do Rio Preto.

SILVA, F.R., SANTOS, R.S., NUNES, M.A. \& ROSSA-FERES, D.C. 2009. Anuros capturados em armadilhas de queda em três agrossistemas no noroeste paulista, Brasil. Biota Neotrop. 9(4): http://www.biotaneotropica.org.br/v9n4/en/abstract?shortcommunication+bn01109042009 (último acesso em 12/06/2010).

SILVA, F.R., PRADO, V.H.M. \& ROSSA-FERES, D.C. 2010. Amphibia, Anura, Hylidae, Dendropsophus melanargyreus (Cope, 1887): Distribution extension, new state record and geographic distribution map. CheckList 6:402-404.

SILVA, R.A., MARTINS, I.A. \& ROSSA-FERES, D.C. 2008. Bioacoustics and calling site in anuran assemblages of open area in the northwest of São Paulo State, Brazil. Biota Neotrop. 8(3): http://www.biotaneotropica.org.br/v8n3/en/abstract?article+bn01608032008 (último acesso em 12/06/2010).

TEIXEIRA, M.G. 2009. Distribuição espacial e temporal da comunidade de anfíbios anuros de um remanescente de mata na região de Botucatu, SP. Dissertação de Mestrado, Universidade Estadual Paulista Júlio de Mesquita Filho, Botucatu.

THOMÉ, M.T.C. 2006. Diversidade de anuros e lagartos em fisionomias de Cerrado na região de Itirapina, sudeste do Brasil. Dissertação de mestrado, Universidade de São Paulo, São Paulo. 
Rossa-Feres, D.C. et al.

THOMÉ, M.T.C., OYAMAGUCHI, H.M. \& BRASILEIRO, C.A. 2007. Amphibia, Anura, Leiuperidae, Physalaemus bokermanni: Distribution extension. Check List 3(1):1-3.

TIBURCIO, I.C.S., LISBOA, B.S. \& HADDAD, C.F.B. 2008. Amphibia, Anura, Cycloramphidae, Macrogenioglottus alipioi: Distribution extension, state of São Paulo and Alagoas, Brazil. Check List 4(4):455-457.

TITON JUNIOR, B., NAVAS, C.A., JIM, J. \& GOMES, F.R. 2010. Water balance and locomotor performance in three species of neotropical toads that differ in geographical distribution. Comp. Bioch. Physiol. A, Mol. \& Integrative Physiol. 156:129-135. PMid:20096361. http://dx.doi.org/10.1016/j.cbpa.2010.01.009

TOLEDO, L.F., ZINA, J. \& HADDAD, C.F.B. 2003. Temporal and Spatial Distribution in an Anuran Community in Municipality of Rio Claro, São Paulo, Brazil. Holos Environ. 3(2):136-149.

TOLEDO, L.F., CASTANHO, L.M. \& HADDAD, C.F.B. 2005. Reconhecimento e distribuição de Leptodactylus mystaceus (Anura; Leptodactylidae) no Estado de São Paulo, Sudeste do Brasil. Biota Neotrop. 5(1): http://www.biotaneotropica.org.br/v5n1/pt/abstract?article+BN00505012005 (último acesso em 8/05/2010).

VASCONCELOS, T.S. \& ROSSA-FERES, D.C. 2008. Habitat heterogeneity and use of physical and acoustic space in anuran communities in southeastern Brazil. Phyllomedusa 7:125-140.

VASCONCELOS, T.S. 2009. Diversidade, padrões espaciais e temporais de anfíbios anuros em uma floresta estacional semidecidual Atlântica, Parque Estadual do Morro do Diabo. Tese de doutorado, Universidade Estadual Paulista Júlio de Mesquita Filho, Rio Claro.

VASCONCELOS, T.S., SANTOS, T.G., ROSSA-FERES, D.C. \& HADDAD, C.F.B. 2010. Similarity of ground-dwelling anuran (Amphibia) composition among different vegetation physiognomies in a Mesophytic Semideciduous Forest from southeastern Brazil. North-West.J. Zool. 6:275-285.

VERDADE, V.K., CASSIMIRO, J. \& RODRIGUES, M.T. 2009. Amphibia, Anura, Cycloramphidae, Zachaenus carvalhoi Izecksohn, 1983 and Z. parvulus (Girard, 1853): Filling gap and geographic distribution map for the genus. Check List 5(4): 755-758.

ZINA, J., SÁ, F.P. \& PRADO, C.A.P. 2010. Amphibia, Anura, Hylidae, Hypsiboas raniceps Cope,1862: Distribution extension. Check List 6(2):230-231. 